University of Louisville

ThinkIR: The University of Louisville's Institutional Repository

Electronic Theses and Dissertations

8-2014

\title{
Bone strength and architecture : pharmacological targeting of CaMKK2 as a method for enhancing bone quality.
}

Zachary James Pritchard

University of Louisville

Follow this and additional works at: https://ir.library.louisville.edu/etd

Part of the Pharmacy and Pharmaceutical Sciences Commons

\section{Recommended Citation}

Pritchard, Zachary James, "Bone strength and architecture : pharmacological targeting of CaMKK2 as a method for enhancing bone quality." (2014). Electronic Theses and Dissertations. Paper 1156.

https://doi.org/10.18297/etd/1156

This Master's Thesis is brought to you for free and open access by ThinkIR: The University of Louisville's Institutional Repository. It has been accepted for inclusion in Electronic Theses and Dissertations by an authorized administrator of ThinkIR: The University of Louisville's Institutional Repository. This title appears here courtesy of the author, who has retained all other copyrights. For more information, please contact thinkir@louisville.edu. 


\title{
BONE STRENGTH AND ARCHITECTURE: PHARMACOLOLOGICAL TARGETING OF CAMKK2 AS A METHOD FOR ENHANCING BONE QUALITY
}

By

Zachary James Pritchard

B.S. Sierra Nevada College 2011

\author{
A Thesis \\ Submitted to the Faculty of the \\ School of Medicine at the \\ University of Louisville \\ In Partial Fulfillment of the Requirements \\ For the Degree of \\ Master of Science \\ Department of Pharmacology and Toxicology \\ University of Louisville \\ Louisville, Kentucky
}

August 2014 
Copyright 2014 by Zachary James Pritchard

All rights reserved 

BONE STRENGTH AND ARCHITECTURE: PHARMACOLOLOGICAL TARGETING OF CAMKK2 AS A METHOD FOR ENHANCING BONE QUALITY

\section{By}

Zachary James Pritchard

B.S. Sierra Nevada College 2011

A Thesis Approved on

June $24^{\text {th }}, 2014$

By the following Thesis Committee

\begin{tabular}{c}
\hline Dr. Uma Sankar \\
\hline Dr. Michael Voor \\
\hline Dr. William Pierce
\end{tabular}


DEDICATION

This thesis is dedicated to my parents and grandparents 


\section{ACKNOWLEDGEMENTS}

I would like to thank first and foremost my mentor Dr. Uma Sankar. Her patience and assistance with me has been far and above than one could ask for. I'd also like to thank the members of Dr. Sankar's lab, Rachel and Cierra. I thank Dr. Voor and his lab for teaching me the equipment required for this project and letting me become part of his lab. I thank Dr. William Pierce for being a part of my thesis committee and want to thank the Dept. of Pharmacology and Toxicology for helping me through this process. 


\begin{abstract}
BONE STRENGTH AND ARCHITECTURE: PHARMACOLOLOGICAL TARGETING OF CAMKK2 AS A METHOD FOR ENHANCING BONE QUALITY

Zachary James Pritchard
\end{abstract}

June $24^{\text {th }} 2014$

Mice lacking or pharmacologically inhibited for calcium/calmodulin-dependent protein kinase kinase 2 (CaMKK2) have enhanced bone mass and microarchitecture. This enhanced bone mass and architecture is due to changes in osteoblast and osteoclast numbers as well as activity. Whether the enhanced bone mass translated to increased bone quality and strength was further explored by developing a method to directly test the trabecular strength of the distal femur. Micro computed tomography (CT)-based measurement of the length of the epiphysis of the distal femur, aided in its removal, allowing the exposure of the trabecular bone volume used to determine the microarchitecture parameters. Following the development of this method, the hypothesis that the lack or inhibition of CaMKK2 results in enhance bone strength as well as bone mass could be tested. Our results then show a strong correlation between the enhanced bone mass and bone strength in animals lacking or acutely inhibited for CaMKK2. 
TABLE OF CONTENTS

Page

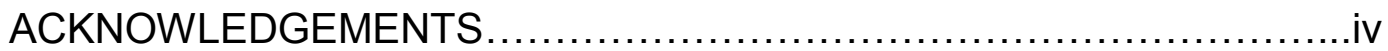

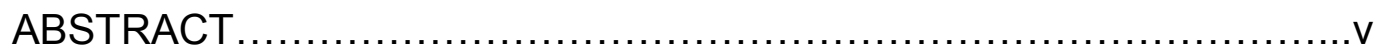

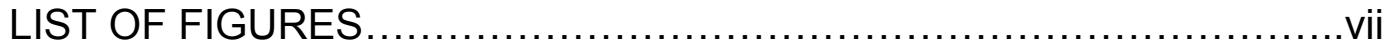

CHAPTER1: INTRODUCTION ..........................................

CHAPTER 2: Distal femur indentation: An improved method for determining

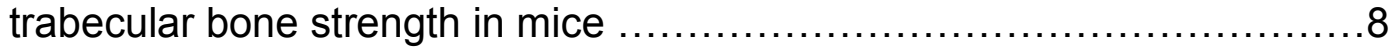

Introduction ..........................................................

Materials and Methods.......................................... 10

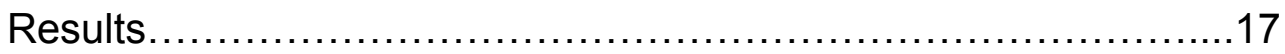

Discussion......................................................... 19

CHAPTER 3: Acute Inhibition or Lack of CaMKK2 Enhances Bone Mass, Microarchitecture and Strength in Adult Mice .............................21

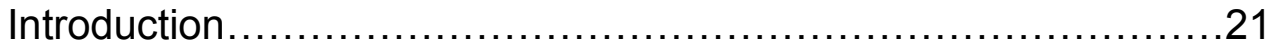

Materials and Methods..............................................

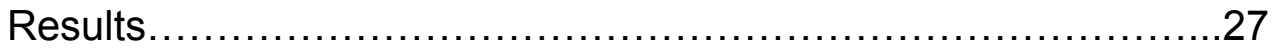

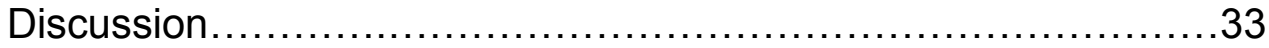

CHAPTER 4: SUMMARY \& DISCUSSION ..............................46

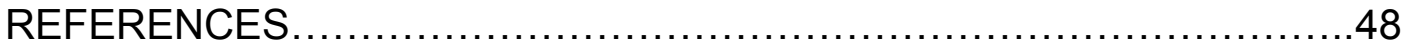

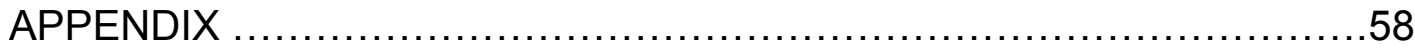




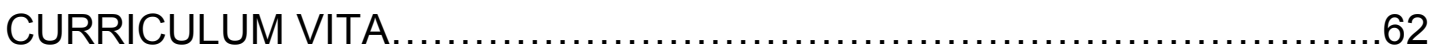




\section{LIST OF FIGURES}

Figure $\quad$ Page

1. Determining the trabecular indentation site using micro-CT imaging........12

2. Femurs mounted prior to epiphysis removal. ............................13

3. Femur prior to indentation, after indentation and representative mechanical

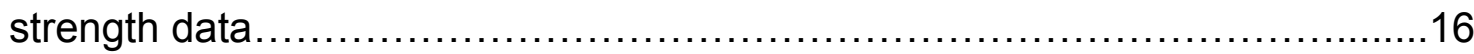

4. Longitudinal cross-sectional images and numerical values accompanying wild type and Camkk2 $2^{-1}$ animals.................................................17

5. Individual epiphysis lengths and trabecular strength data...................19

6. Twelve week old male Camkk2/ mice possess elevated trabecular bone volume fracture and improved microarchitecture ............................... 38

7. Higher trabecular bone strength in younger Camkk2 $2^{-/}$mice. ................39

8. Lack of CaMKK2 results in increased cortical bone bending strength and middiaphysis geometry without affecting bending stress.

9. STO-609 administration stimulates trabecular bone formation in thirty-two week old adult male mice.

10. Increased trabecular bone volume and enhanced microarchitecture is sustained in adult mice lacking or acutely inhibited for CaMKK2.

11. Higher trabecular and cortical bone strength in adult Camkk2 ${ }^{-/}$and STO-609treated WT mice. 


\section{CHAPTER 1 \\ INTRODUCTION}

Osteoporosis is a pathological condition wherein decreased bone strength increases the risk of fracture. Bone strength is a term that intertwines bone quality and bone density. Bone quality refers to the material and architectural properties of the bone while bone density simply measures the amount of bone present. Osteoporosis is widely known to accompany aging; specifically in elderly women. Hormonal changes are the most common contributor of osteoporosis, with post-menopausal women being the prime example. Osteoporosis, however, can also be an unintended consequence of treating other illnesses. Patients taking glucocorticoids are an example, often unintentionally developing osteoporosis [1, 2]. Osteoporosis is a leading risk factor for a variety of problems, including fracture. Fracture events range greatly, from simple transverse fractures to convoluted comminuted fractures. Hip fractures are credited with the lion's share of complications and many treatment options are directed towards these types of fractures in particular [3]. The development of clots and pneumonia are common after such breaks and are some of the leading problems faced by hip fracture patients. These fractures are often exacerbated by other confounding conditions and increase a patient's risk of mortality after fracture [4]. Thus prevention of fractures remains a principal objective in assisting osteoporotic patients. Progress towards this goal is predominantly achieved through maintaining and improving bone strength. 
Addressing osteoporosis requires understanding the underlying processes that dictate the continuous upkeep of the skeleton. Tasked with this are a variety of different cells, the two that have garnered the most focus are the osteoblasts and osteoclasts. Osteoblasts concern themselves with depositing osteoid and facilitating the mineralization of our bones. Osteoclasts, on the other hand, resorb bone in response to a complex signaling pathway. The osteoclasts and osteoblasts each regulate the other and overall, this dynamic process is deemed "bone remodeling" [5]. The two types of cells work in concert to repair, maintain, and adjust the skeleton. This process balances itself accordingly throughout one's lifetime to meet the body's demands. Osteoporosis describes a change in this balance where deposition of bone falls below that of its removal. This results in net losses of material; generating bone weaknesses resulting in risks to health. Treating osteoporosis has been concerned with manipulating bone remodeling. Both lifestyle changes and pharmacological intervention are often part of treating osteoporosis.

Developing positive life style habits have been important to decreasing the likelihood of falls. Mechanical stimulation from exercise positively influences bone remodeling. The resulting influence has both qualities of anabolic and ant-catabolic qualities treatments[6]. As activity levels decrease with age, maintaining some level of activity ensures a slower rate of bone loss, and potentially reverses some of the deleterious effects of osteoporosis. Supplementing exercise increases one's awareness, leading to the prevention of falls. Falls can be avoided with simple exercises that strengthen and improve gait 
and balance [7] or avoiding seasonal hazards such as ice. However, such an increased sense of mobility can also lead to an increased number of falls due the increased exposure to risk, thus giving the perception of exercise being ineffective. Positive changes in diet have also been shown to protect against osteoporosis. Calcium supplements or increased consumption of calcium rich food is often prescribed as an aid against osteoporosis. This tactic should not be limited to the onset of osteoporosis but may be best to act decades in advance. Dietary measures such as increasing milk consumption in children and adolescents appear to have a protective effect against the onset and development of osteoporosis [8]. Vitamin D is often given also as a treatment in addition to calcium, maximizing calcium absorbed. Both have been shown to decrease prevalence of fractures [9]. Behavioral modifications are but one aspect of managing osteoporosis and are often used alongside pharmacological choices to potentiate long term success.

The primary approaches for pharmacologically addressing osteoporosis include both anti-resorptive and anabolic treatment strategies. Anti-resorptive treatments are chiefly tasked with restricting bone reabsorbed during bone remodeling, resulting in a net gain in bone deposited. Bisphosphonates (BPs) are the most common anti-resorptive option. BPs act primarily through binding to calcium and promoting osteoclast apoptosis when encountered, which in turn lowers the volume of bone removed, allowing more bone to remain [10]. Antiresorption can be accomplished by alternate routes, such as interrupting signal molecules to osteoclasts. An example for this tactic is Denosumab. 
Denosumab is an inhibitor of nuclear factor kappa $b$ ligand (RANKL). RANKL has a role in the maturation and propagation of osteoclasts which, if interrupted, results in a net reduction in bone resorbed [11]. Manipulating bone remodeling in the resorptive manner however eventually slows the remodeling process and leads to decreased bone tissue quality. The anabolic option for treatment is more appealing because it avoids this limitation.

At present, there is only one approved option for anabolic treatment of osteoporosis. Para-thyroid hormone (PTH) or teriparatide (TPTD) has a stimulatory effect on bone remodeling and encourages an accelerated rate of remodeling which can result in an increase in bone quality.

PTH does stimulate bone remodeling, however this influences the entire bone remodeling system. That is both bone deposition and bone removal are increased. With intermittent use, PTH can be used to stimulate osteoblast activity as it binds to osteoblasts, and without continued presence of PTH osteoclastogenesis is not upregulated allowing for bone deposition and limited amounts of bone resorption. If PTH is allowed to have continuous high levels then bone removal would be upregulated also. [12].

Current options for the treatment of osteoporosis help reduce the risk of fracture and, in some cases, actively enhance bone quality. However, antiresorptive and anabolic treatments have a variety of limitations. For example, there are a variety of adverse effects ranging from gastrointestinal upset to unfavorable methods of delivery. These reduce patient compliance s[13]. Potential treatment options of osteoporosis would need to limit the amount of 
side effects and allow for higher patient compliance. Such treatments would be screened to also include both anabolic and anti-catabolic qualities. One such option may lie with the serine-threonine kinase CaMKK2.

Calcium/calmodulin-dependent protein kinase kinase two (CaMKK2) is a member of the CaMK cascade and is activated with the release of intercellular calcium. CaMKK2 and phosphorylates CaMK1 or CaMK4 and has roles in regulating energy balance, acting through AMPK [14],. This serine-threonine kinase family has members that are ubiquitously expressed such as CaMK1 and CaMK2, but CaMKK2 itself tissue-restricted [15]. Its locations include different areas of the brain and lower levels of CaMKK2 are expressed in the bone, spleen and lungs. Expression and the ablation thereof in murine models have been linked with impairment of memory in males [16] and susceptibility to schizophrenia [17]. These links to unfavorable conditions or possible adverse effects of targeting CaMKK2 are paired with distinct benefits. These potential worrisome drawbacks may be avoided with conditional knockout models or drug intervention.

Mice deficient in CaMKK2 appear to be protected from diet induced obesity. This protection extends to glucose intolerance and insulin resistance derived from influencing macrophages activation and response [15]. Hematopoietic stem cell (HSC) numbers were decreased along with a decreased total of bone marrow cells. However these reduced populations of HSCs suffer no difference in proliferation or survival [18]. Animals deficient in CaMKK2 show an immediate visual difference in their skeletal system; the long bones appear 
paler than wild type animals. This difference investigated further revealed significant differences in bone cells. Osteoblasts and osteoclasts populations were inversely altered; osteoblast populations grew whilst osteoclast populations declined. Osteoclasts additionally displayed fewer nuclei and decreases in their relative size. Changes present in cellular populations were joined with significantly increased bone volume. Altering naturally occurring ratios of osteoblasts and osteoclasts appears to be responsible for promoting the observed phenotype. Dramatic changes in bone volume signaled that targeting CaMKK2 may be an option for protecting against osteoporosis. Experiments utilizing genetic ablation can often be reproduced in slight with pharmacological intervention. Inhibition of CaMKK2 can be achieved with the selective inhibitor STO-609 [19]. This compound is selective not specific; and at higher concentrations can inhibit other CaMK members. STO-609 was used successfully to evoke similar effects in wild type animals that were observed in knockouts. These effects were reflected in both osteoblast and osteoclast populations, and offered protection from ovariectomy induced osteoporosis [20]. These effects indicated the utility of acutely inhibiting CaMKK2 without the inherent weaknesses belonging to whole specimen knockouts. Adverse effects resulting from STO-609 use have yet to be fully described and are being explored alongside practical applications.

Managing osteoporosis has been both fruitful and troublesome. Many risks stemming from it can be mitigated with behavioral and pharmacological intervention. With the options available, any anabolic approach and the 
advantages therein would be valuable in managing osteoporosis. The goal of the studies described herein is to investigate whether the acute pharmacological inhibition of CaMKK2 by STO-609 significantly increases bone quality in addition to having positive impacts on bone volume, resulting in an increase in bone strength. 


\section{CHAPTER 2}

\section{DISTAL FEMUR INDENTATION: AN IMPROVED METHOD FOR DETERMINING TRABECULAR BONE STRENGTH IN MICE}

\section{Introduction}

Bones are responsible for a variety of functions ranging from support and scaffolding and as levers for movement. Hence, the bone must be strong and capable of supporting the organism. The demands on each organism's bones can be similar but unique, depending on the evolutionary adaptation [21, 22]. Different approaches have been developed to determine the strength of bone. Closely related to other material sciences, compression, tensile and torsional strength are among the different mechanical properties that are tested in bone $[23,24]$. To effectively test these properties, different types of bone can be used to model certain situations. Human vertebrae, for example, can be loaded axially in compression to simulate how the bone will be loaded in vivo.

X-ray and micro-computed tomography (CT) imaging have been used in conjunction with mechanical testing to obtain a full picture of the microarchitectural and biomechanical properties of the bone [25]. Mechanical 
properties of the bone can be predicted from its microarchitecture. Thus, virtual inspection performed in conjunction with biomechanical testing can validate any potential correlations between image data and mechanical strength.

Because genetically modified mouse models are highly valuable tools in understanding the molecular pathways regulating bone biology, robust methods to test mechanical strength of murine long bones are critical. Whereas methods to test cortical bone strength such as three- and four-point bending tests are welldeveloped, assays that will effectively test trabecular bone strength in murine long bones are still lacking. This study is concerned with determining the strength of murine trabecular bone in the femur. Working with murine bones offers unique challenges, given the small size of mouse skeletons. Femurs were chosen because of their size and the relative ease in the way they can be handled. Moreover, the femur offered a prime area to image using micro-CT while also being a possible site for mechanical testing. Besides, the small size of murine femurs precludes the need to sub-sample cubes, as is done with larger animals [26]. In this study, we developed a novel method of using reliable micro$\mathrm{CT}$ imaging as a basis for determining mechanical strength of murine trabecular bone. We used a murine genetic model with predictable differences in microarchitecture to validate this technique [27]. 


\section{Methods}

Mice

Five month old male wild-type $(\mathrm{WT}, \mathrm{n}=5)$ and $\operatorname{Camkk}^{/-}(\mathrm{n}=5)$ mice were housed in the Baxter II Vivarium at the University of Louisville. The mice were under a 12-hr light and dark cycle, with food and water provided ad libitum. All care and experimental procedures were performed according to University of Louisville (UofL) Institutional Animal Care and Use Committee protocols and in compliance with NIH guidelines on the use and care of laboratory and experimental animals.

Micro-computed tomography (Micro-CT)

Micro-CT imaging was performed at the University of Louisville Orthopaedic Bioengineering Lab with a high resolution CT scanner (Actis HR225150; BIR, Lincolnshire, IL, USA). Individual or groups of femurs were imaged at a voxel resolution of $7 \mu \mathrm{m}$. An approximate length of $5.25 \mathrm{~mm}$ was imaged. Transverse images were processed using both two-dimensional (Image J, NIH Image, Bethesda, MD) and three-dimensional software (VG Studio Max, Volume Graphics, Heidelberg, DE).

Removal of epiphysis

Transverse micro-CT image stacks, spanning from the first appearance of a condyle to the base of the epiphysis, were used to determine the length of the growth plate at the distal end of the femur. With an isotropic voxel resolution of 7 
$\mu \mathrm{m}$ per slice, the numbers of slices encompassing the region were counted (Figure 1). The epiphysis/metaphysis junction, appearing as a cross with four chambers on the micro-CT image (slice 177, Figure 1), represents the region where the trabecular bone begins and is the site of the indentation test. As shown in Figure 1, the length of the epiphysis was then calculated in $\mathrm{mm}$ 


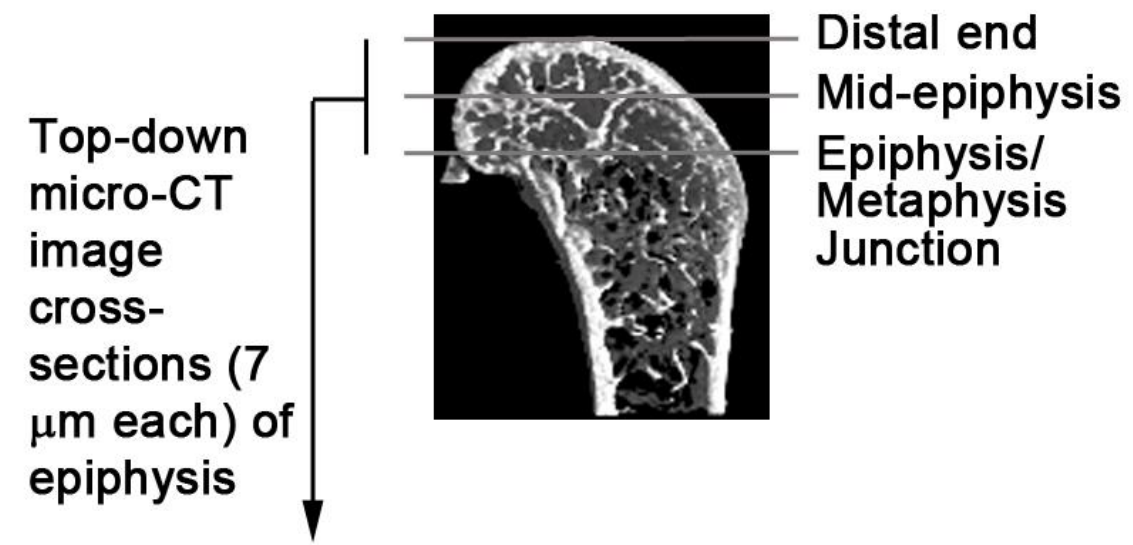

Distal end $=$ Slice $25 \quad$ Middle $=$ Slice 111 Junction $=$ Slice 177
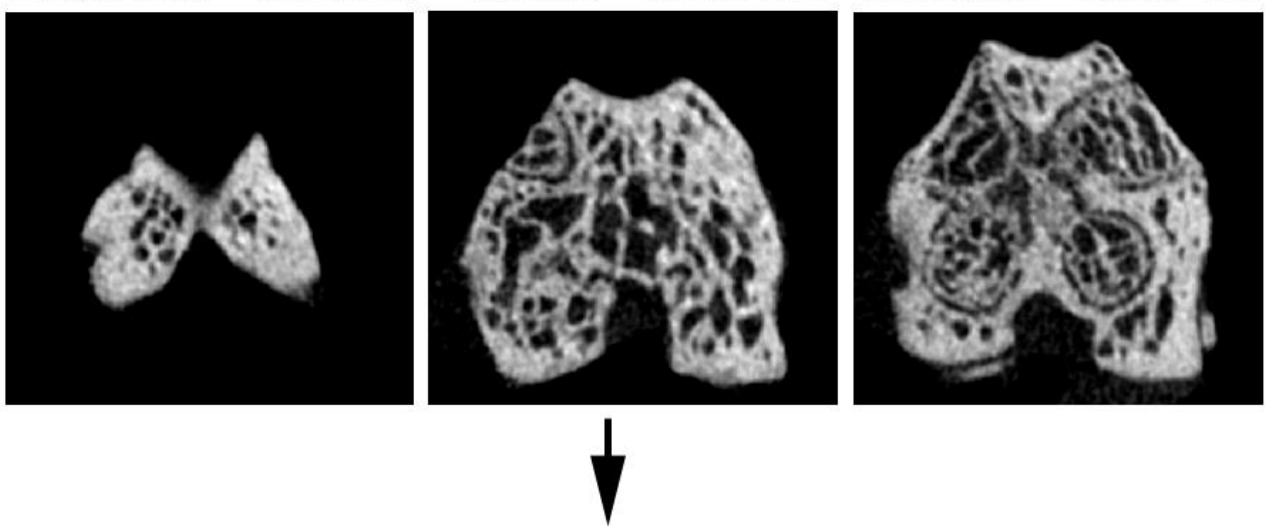

Each cross-section or slice $=7 \mu \mathrm{m}$ thickness 177 slices $=7 \times 177=1239 \mu \mathrm{m}=1.24 \mathrm{~mm}$ Cutting length $=1.24 \mathrm{~mm}$

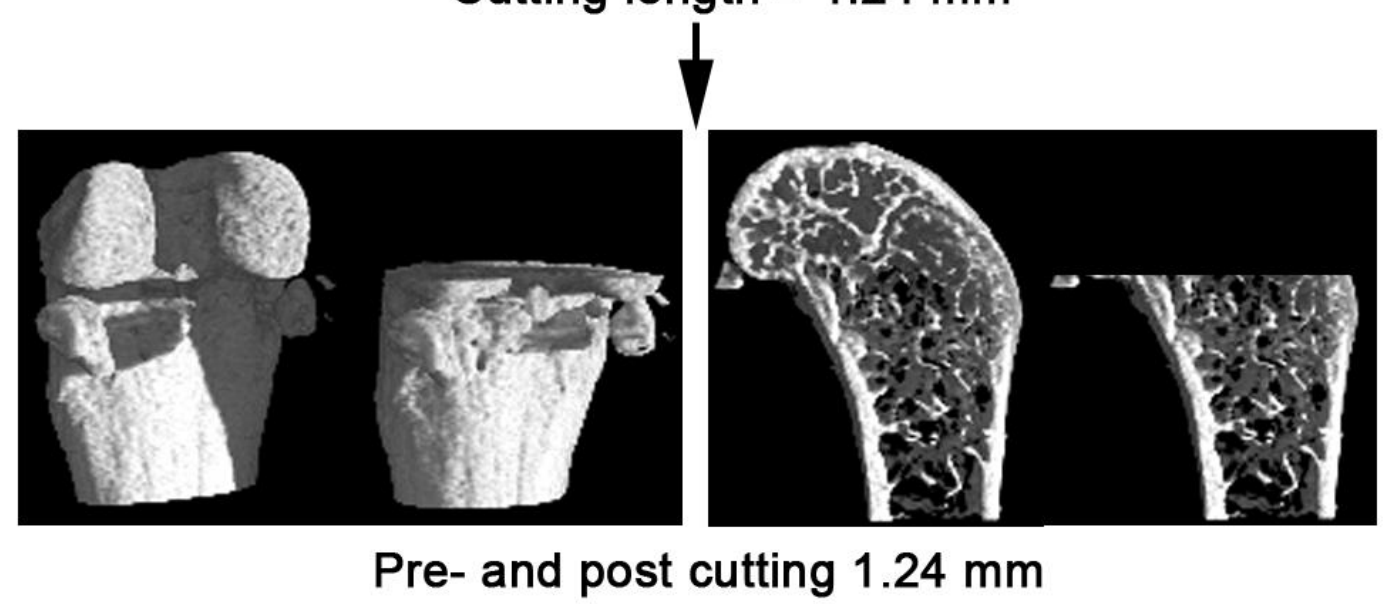




\section{Figure 1. Determination of the indentation site by micro-CT imaging.}

Transverse $\mu \mathrm{CT}$ images were reconstructed into 3-dimensional images (top).

Cross-sectional $\mu \mathrm{CT}$ images were counted and converted into a distance in $\mathrm{mm}$ (middle). This distance was used to determine the amount of bone material to be removed for mechanical testing (bottom).

In order to remove the epiphysis the femurs needed to be properly mounted. Using a two part epoxy mix (Loctite ${ }^{\circledR}$ Epoxy Quick Set ${ }^{\mathrm{TM}}$, Westlake, $\mathrm{OH}, \mathrm{USA}$ ), each femur was mounted atop and centered on a socket headed \#10 cap screw (Fastenal, USA). Using an appropriate amount of epoxy is critical for proper mounting, and varies based on the sample. Enough epoxy must be used to create the base that will surround the femur past the metaphysis and even up to the condyles (Figure 2A). Samples were allowed to cure for 24 hours.
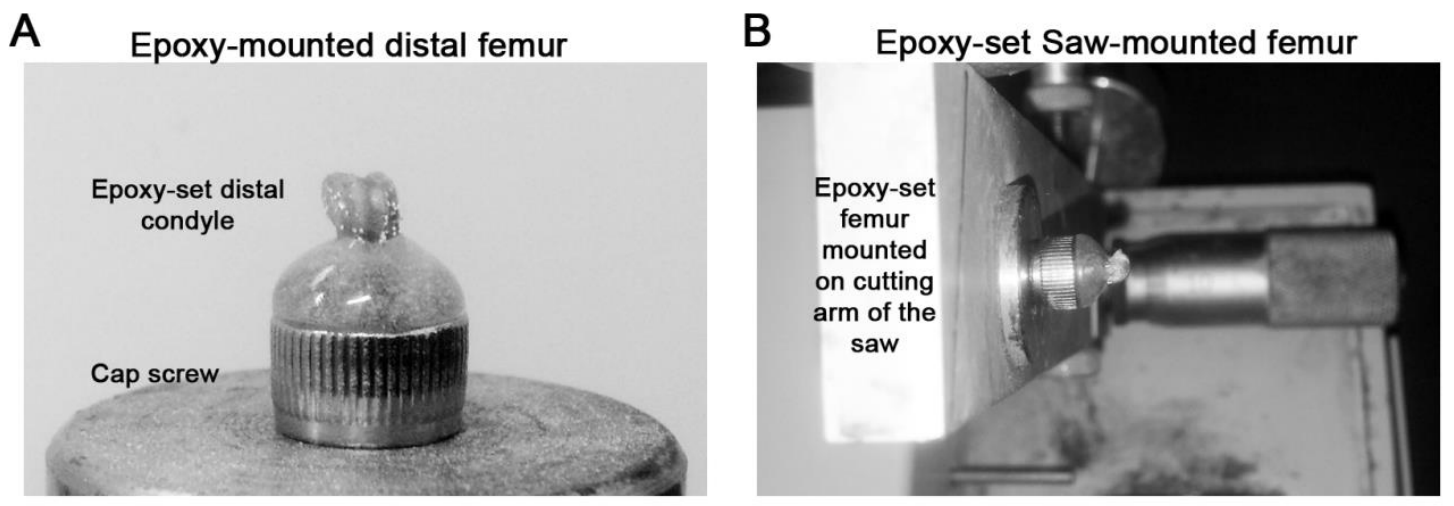

Figure 2. Femurs mounted prior to epiphysis removal. (A) Femurs were mounted atop a \#10 socket headed screw with Loctite® Epoxy and cured for 24 hours.(B) Calculated lengths of the epiphysis were then removed using an Isomet ${ }^{\circledR}$ diamond sectioning saw 
Removal of the epiphysis was done with a diamond sectioning saw (Isomet $\AA$, Lake Bluff, IL USA). Because the saw is calibrated in inches, we converted the epiphysis length measurement from $\mathrm{mm}$ to inches. The epoxy-set femur was mounted on the diamond saw and advanced according to the calculated epiphysis length in inches (Figure 2B). Removal of the epiphysis was carefully performed to limit stress. A quick cutting speed was used to reduce the amount of stress the femur underwent, and to avoid disruption of the epoxy mount.

Indentation

Indentation of distal femoral trabecular bone was performed via blunt instrumentation. The indenter used to penetrate the cancellous bone was machined from a $3 / 4$ inch bolt into a blunt cylindrical surface, and measured $3 \mathrm{~mm}$ in length and $1 \mathrm{~mm}$ in diameter (Figure $3 \mathrm{~A}$ ). The indenter produced $1.27 \mathrm{MPa}$ of compressive stress for every Newton $(\mathrm{N})$ of force that was applied.

Immediately following the removal of epiphysis, the femur was centered on a servo-hydraulic load frame (Model 858 Bionix, MTS Corp., Eden Prairie, $M N)$. Due to the curvature of the femur, it is possible for the indenter to encounter the cortical bone of the metaphyseal flare during indentation. Hence, proper alignment of the indenter was important. Centering the indenter is intuitive, but aligning the indenter to be slightly anterior of dead center is required. 
The lowest point during the first $1.5 \mathrm{~mm}$ of penetration (force $\mathrm{X}$ displacement in millimeters), as shown in the graphs (Figure 3B), is determined to be the failure of trabecular bone upon indentation. Indentation was performed by applying force axially at a rate of $1 \mathrm{~mm} /$ minute. The maxium depth of the test was the full $3 \mathrm{~mm}$ length of the indenter. However, the first $1.5 \mathrm{~mm}$ (1.5 minutes) is the accepted region of testing, as it measures the strength of the trabecular microarchitecture (Figure 3Bi). Anything further than than that is disregarded. Additionally, any ambigiuous local minima were also disregarded (Figure 3Bii). Data in this case may be suspect due to architechural interference, such as contacting the cortex. . Visual confirmation of proper indentation of individual femurs should be performed by micro-CT (Figure 3C) 
A

Mounted femur prior to indentation

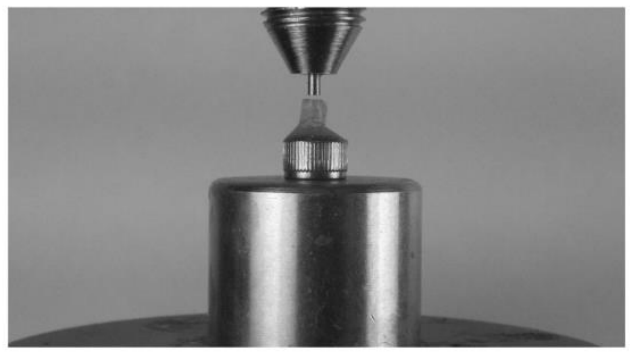

$\mathrm{Bi}$

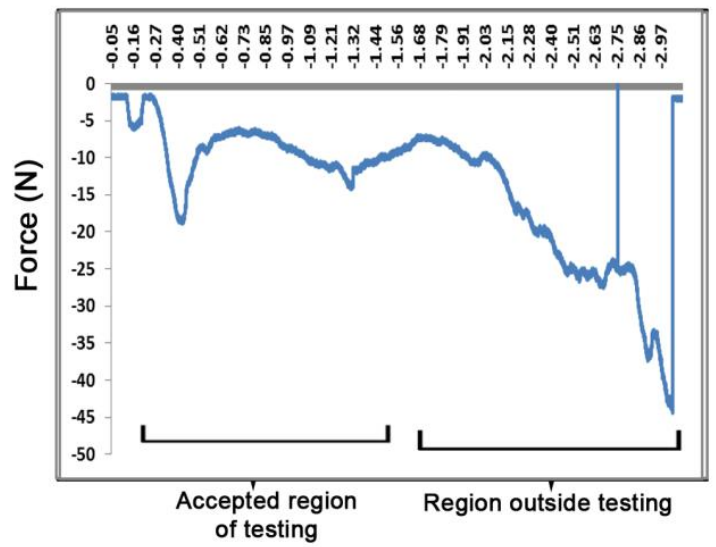

C Micro-CT crosssections before and after indentation
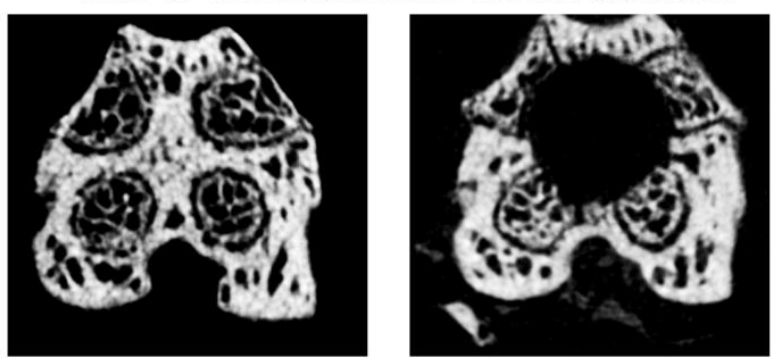

Bii

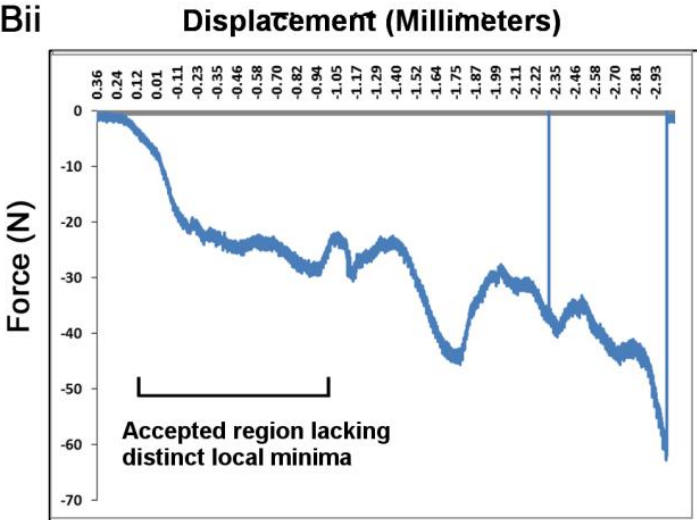

Figure 3. Femurs prior to indentation, after indentation and representative mechanical strength data. (A) Femur with the epiphysis removed and placed before indentation. The Indenter used is also shown. (B) Strength data gathered from both acceptable and unacceptable test results. The distinct local minima in Bi clearly show a failure in trabecular strength. Ambiguous data graphs as shown in Bii do not easily discern a point of failure. (C) Micro-CT cross-sectional images showing the femur after indentation. 


\section{Results}

The purpose of this study was to develop a robust methodology to measure trabecular bone strength in murine femurs. We hypothesized that slicing off the distal femur epiphysis at the exact epiphysis-metaphysis junction would enable the exposure of the trabecular bone, enabling the accurate measurement of its strength. To test this idea, we used long bones from mice that are genetically knocked out for the expression of $\mathrm{Ca}^{2+} /$ calmodulin dependent protein kinase kinase 2 (CaMKK2; Camkk2 ${ }^{-/}$mice), that possess enhanced trabecular bone volume in their long bones when compared to age- and sex-matched WT mice,[27] (Figure 4, Table1). Micro-CT images revealed that the trabecular bone in Camkk2 $2^{-/}$distal femurs were most concentrated just beneath the growth plate (Figure 4 - book slice image), presenting us with the right model system to test our hypothesis.

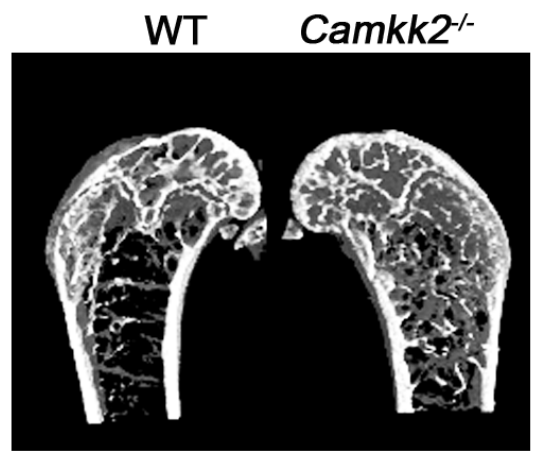

Table 1: Microarchitecture Measurements

\begin{tabular}{lccc}
$\begin{array}{l}\text { Microarchitecture } \\
\text { Parameters }\end{array}$ & WT & Camkk2 $^{\%}$ & $p$-value \\
\hline BV/TV $(\%)$ & $19.8 \pm 3.9$ & $29.4 \pm 5$ & 0.003 \\
Tr.Th. $(\mu \mathrm{m})$ & $38.4 \pm 4.6$ & $41.5 \pm 3.1$ & 0.15 \\
Tr.Sp. $(\mu \mathrm{m})$ & $158.3 \pm 3.11$ & $102.1 \pm 16.8$ & 0.0002 \\
\hline
\end{tabular}


Figure 4. Longitudinal cross-sectional images and numerical values accompanying wild type and Camkk2/- animals. Shown left are longitudinal book slices of wild type and knockout femurs. The distribution of bone shows more trabecular architecture relegated towards the distal end. (Table 1) Detail the mean values for bone volume fraction, trabecular thickness, and trabecular separation along with the $p$-value with each parameter.

Sequential stacked transverse micro-CT sections of the growth plate were used to determine the length of the distal epiphysis of individual femurs from 5 month-old WT and Camkk2/- mice (Figure 5 and Table 2). These data were then converted to length in $\mathrm{mm}$ from the distal tip of the femurs, enabling the determination of accurate cutting lengths (Table 2). Results from the blunt indentation performed thereafter showed markedly higher trabecular compression strength in Camkk2 $2^{-/}$distal femurs, compared to WT (Table 2, Figure 5). 
Table 2: Individual Epiphysis Length and Tabecular Strength Measurements

\begin{tabular}{ccccc}
\hline & \multicolumn{2}{c}{ WT } & \multicolumn{2}{c}{ Camkk2 $^{\%}$} \\
\hline $\begin{array}{c}\text { Epiphysis } \\
\text { Length (mm) }\end{array}$ & $\begin{array}{c}\text { Strength } \\
(\mathrm{MPa})\end{array}$ & $\begin{array}{c}\text { Epiphysis } \\
\text { Length (mm) }\end{array}$ & $\begin{array}{c}\text { Strength } \\
(\mathrm{MPa})\end{array}$ \\
\hline & 1.211 & 42.96 & 1.12 & 64.34 \\
& 1.274 & 35.33 & 1.155 & 78.41 \\
& 1.239 & 31.02 & 1.113 & 42.18 \\
& 1.239 & 46.64 & 1.323 & 52.24 \\
& 1.274 & 51.13 & 1.211 & 50.67 \\
\hline Mean & 1.2474 & 41.42 & 1.1844 & 57.57 \\
Stdev & 0.0268 & 8.20 & 0.0866 & 14.08 \\
\hline
\end{tabular}

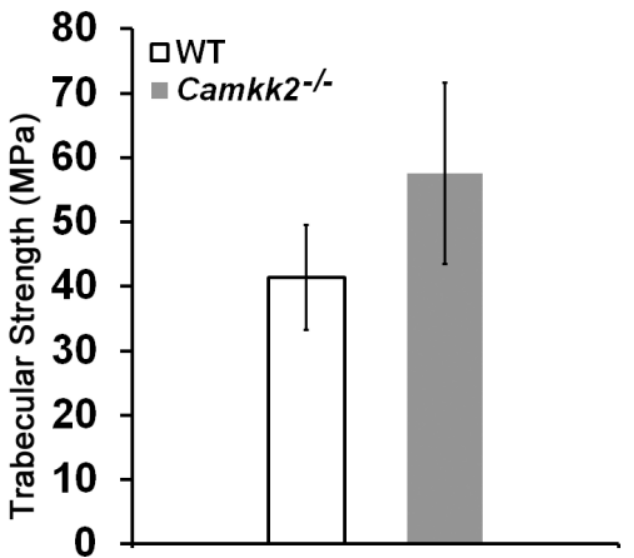

Figure 5. Individual epiphysis lengths and trabecular strength data. (Table 2) lists individual lengths of the epiphysis and the strength data of each femur. Shown right are the mean values with standard deviation shown. The data here have a p-value of 0.058 .

It is important to measure individual cutting lengths using micro-CT imaging, rather than relying on an average value. As indicated in Table 2, the average values usually exceed individual epiphyseal lengths. This will lead to cutting too far below the growth plate and missing the area of interest - i.e., epiphysis-metaphysis junction where the trabecular bone is concentrated. If an average cutting length is utilized to expose the trabecular bone, the differences in trabecular compression strength may no longer be discernable.

\section{Discussion}

This paper details a method for determining the strength of trabecular bone in the distal femur. Accurate measurement of the region of interest through calculations based on micro-CT imaging, aided in the removal of 
epiphysis and exposure of the trabecular bone at the base of the growth plate (Figure 1). This enabled the direct testing of the correlation between microarchitecture and trabecular strength. . With bone distributed more towards the growth plate, any excess removal of the epiphysis would be reflected in the strength data.

The strength data also can give clues as to how well the technique worked. With acceptable local minima, such as Figure 4Bi, a strong correlation can be observed between trabecular microarchitecture (bone mass) and trabecular strength. With the data set presented in Figure 5 and Table 2, this correlation has an $r^{2}$ coefficient of 0.654 when adjusted for epiphyseal length. Thus in conclusion, the method described herein offers an improved method for determining trabecular strength in the distal femurs of mice. 
CHAPTER 3

\section{ACUTE INHIBITION OR LACK OF CAMKK2 ENHANCES BONE MASS, MICROARCHITECTURE AND STRENGTH IN ADULT MICE}

\section{Introduction}

The skeleton consists of a multifunctional tissue that protects internal organs, facilitates coordinated motion through muscle contraction and maintains physiological $\mathrm{Ca}^{2+}$ homeostasis [28]. Osteoporosis, a severe age-related bone disease that increases the risk of fragility fractures is characterized by the loss of skeletal mass, diminished microarchitecture and lower tissue strength [29, 30]. Bone structure and material composition determines its strength, and a number of environmental, hormonal, metabolic, and genetic determinants during growth and aging affect the structure and tissue integrity of the bone [31-33]. The overall skeletal strength is determined by overall bone size, cortical bone porosity and tissue strength as well as trabecular bone volume fraction, number, connectivity, thickness, separation and tissue strength [31, 34]. Loss of trabecular connectivity is one of the early events associated with age and hormone-loss associated bone loss in humans [35].

The highly porous trabecular bone is found at the proximal and distal ends of long bones as well as in vertebral bodies and provides supporting strength to 
these weight bearing bones [31]. The microarchitecture and biomechanical properties of trabecular bone are optimized through active bone remodeling initiated by osteocytes and executed by osteoclasts and osteoblasts (reviewed in [32]). The remodeling process is in turn influenced by hormonal, paracrine and autocrine signals such as estrogen, testosterone, parathyroid hormone (PTH), insulin-like growth factor 1 (IGF-1), wingless (Wnt), as well as biomechanical stresses [35-38]. Moreover, studies using global and tissuespecific gene knockout murine models have uncovered specific genetic influences on bone mass and/or strength [39-54].

We recently identified novel roles for $\mathrm{Ca}^{2+} /$ calmodulin (CaM)-dependent protein kinase kinase 2 (CaMKK2) in the anabolic and catabolic pathways of bone remodeling [55]. CaMKs are a family of multifunctional serine/threonine protein kinases that includes CaMKK1 $(\square)$, CaMKK2 ( $\square$ ), CaMKI, CaMKII and CaMKIV [56-58]. The CaMK signaling cascade is initiated by transient increases of intracellular $\mathrm{Ca}^{2+}$, that are immediately sensed by the ubiquitous $\mathrm{Ca}^{2+}$ receptor CaM [14]. The upstream kinases, CaMKK 1 and 2 are activated trough $\mathrm{Ca}^{2+} / \mathrm{CaM}$ binding and in turn phosphorylate CaMKs I and IV on a critical threonine residue leading to their activation. In addition, CaMKK2 (not CaMKK1) phosphorylates and activates adenosine mono-phosphate activated protein kinase (AMPK) to coordinate cellular energy balance $[59,60]$.

CaMKK2 is tissue-restricted and its loss protects mice from diet-induced obesity, insulin resistance and inflammatory responses [59,61, 62]. Loss of CaMKK2 through global gene deletion positively influences osteoblasts and 
negatively affects osteoclasts, resulting in a net increase of bone mass [55]. Moreover, pharmacological inhibition of CaMKK2 activity using its selective, cellpermeable pharmacological inhibitor STO-609 in wild type (WT) bone marrow (BM) cells accelerates osteoblast differentiation while inhibiting osteoclasts in vitro $[55,63]$. Furthermore, STO-609 treatment protects mice from ovariectomyinduced bone loss by stimulating osteoblasts and inhibiting osteoclasts [55]. The consequence of these opposing effects of the absence of CaMKK2 on bone cell biology is a net increase in trabecular bone mass, that aids in protection from osteoporosis. However, whether this increased trabecular bone mass translates into improved weight bearing capacity and biomechanical properties are outstanding questions.

We hypothesized that the lack and/or acute pharmacological inhibition of CaMKK2 results in enhanced trabecular bone mass as well as improved bone architecture and strength. Our objective for the current study was to assess the trabecular bone microarchitecture as well as trabecular and cortical bone strength in the long bones of Camkk2 $2^{-/}$mice. We also aimed to determine whether the acute inhibition of CaMKK2 using STO-609 would trigger new bone formation in adult WT male mice that are thirty-two week old, an age at which their trabecular bone mass is in a maintenance phase and/or beginning to decline [64]. If so, we further wanted to evaluate whether the microarchitecture and biomechanical strength parameters indicate enhanced bone volume, trabecular properties and strength in STO-609 treated adult mice. 


\section{Materials and Methods}

Mice: WT and Camkk2- mice (C57BL/6 background) were housed in the University of Louisville (UofL) Baxter II Vivarium under a 12-h light, 12-h dark cycle. Food and water were provided ad libitum. All care and experimental procedures were performed according to UofL Institutional Animal Care and Use Committee protocols and in compliance with NIH guidelines on the use and care of laboratory and experimental animals. Twelve or thirty-two week old male mice were used in these experiments.

STO-609 treatment of mice: STO-609 was purchased from TOCRIS Bioscience (Ellisville, MO, USA) and prepared as mentioned previously [55]. Tri-weekly intraperitoneal (i.p.) injections of $200 \square$ l per mouse of either saline $(n=9)$ or 10 M STO-609 $(\mathrm{n}=13)$ were administered into thirty-two week old WT male mice for 6 weeks. Long bones were harvested and femurs were utilized for microcomputed tomography $(\mu \mathrm{CT})$ and microarchitecture analyses as well as for the assessment of cortical and trabecular bone strength. Thirty-two week old male Camkk2 ${ }^{-/}$mice $(n=6)$ were used as controls for microarchitecture and strength analyses.

Dynamic bone histomorphometry: Seven and two days before euthanasia, the saline and STO-609 treated mice were injected via i.p. with calcein $(5 \mathrm{mg} / \mathrm{ml})$ and alizarin red $(15 \mathrm{mg} / \mathrm{ml})$ respectively, at $100 \mu \mathrm{l}$ per mouse. Mice were euthanized 2 days after the alizarin red injection, and decalcified histology and dynamic histomorphometry were performed on tibiae that were fixed in $70 \%$ ethanol. 
Histomorphometric analysis was performed by a blinded observer using BioQuant OSTEO 2010 software (BioQuant Image Analysis Corporation) and standard parameters [65].

Micro-CT Imaging: Micro-computed tomography $(\mu \mathrm{CT})$ imaging was performed on a high resolution CT scanner (Actis HR225-150; BIR, Lincolnshire, IL, USA) located at the University of Louisville Orthopaedic Bioengineering Lab. Up to 3 femurs were imaged simultaneously at an isotropic voxel resolution of $7 \mu \mathrm{m}$. Transverse images of femurs covering a length of approximately $5.25 \mathrm{~mm}$ were processed individually for each femur using a combination of two-dimensional (ImageJ, NIH Image, Bethesda, MD) and three-dimensional (VG Studio Max, Volume Graphics, Heidelberg, DE) imaging software. Trabecular architectural properties including bone volume fraction, trabecular thickness and separation were determined for a standardized region in the distal femoral metaphysis.

Mechanical testing: After the femurs were imaged in the $\mu \mathrm{CT}$ scanner, they were prepared for mechanical testing to determine cortical bone strength using threepoint bending and cancellous bone strength using blunt indentation. A servohydraulic load frame was used for both mechanical tests (Model 858 Bionix, MTS Corp., Eden Prairie, MN).

Three-point-bending was performed by placing the femur across an $8 \mathrm{~mm}$ wide support span and loading vertically with a rounded knife edge at the center of the span contacting the anterior surface of the femoral diaphysis. The loading was applied at a displacement rate of $1 \mathrm{~mm} / \mathrm{min}$ until the femur failed. The 
amount of force, F, applied was recorded and the magnitude of the bending moment at the point of failure could be calculated as: $M=\frac{1}{4} * F * 8 \mathrm{~mm}$. Threepoint-bending typically resulted in the femur breaking into two pieces, a proximal section and a distal section with an oblique or transverse fracture occurring under the center contact point.

The resulting distal section of the femur was then prepared for a blunt indentation test to measure the strength of the cancellous bone material in the distal part of the femoral metaphysis. The distal femur was mounted in the head of a \#10 cap screw using two-part epoxy with the condyles exposed and the shaft of the femur oriented along the axis of the screw (Figure 2A). This provided a means to trim the end off of the femur, exposing the sub-epiphyseal cancellous bone of the distal metaphysis. The screw was mounted in a low-speed diamond sectioning saw and the blade was advanced to coincide with the position of the epiphysis as observed on $\mu \mathrm{CT}$ images taken previously. After a flat surface perpendicular to the shaft axis was exposed, the screw/bone combination was positioned under the blunt indenter tip fixed to the actuator of the MTS machine. The exposed cancellous surface was centered under the indenter and the tip was advanced at a rate of $1 \mathrm{~mm} / \mathrm{min}$. The displacement and compressive force were recorded. The depth of indentation was limited to $1.5 \mathrm{~mm}$ to avoid contact of the indenter tip with the cortical wall of the distal metaphysis.

Equations Used with Mechanical Testing: The maximum applied loads in each type of test can be used as a measure of the cortical and cancellous bone strength, but, to determine the true tissue strength, some normalization is 
required. The three-point-bending failure moment can be converted to a maximum tissue stress using the following equation: $\sigma_{b}=\frac{M y}{I} ;$ where $\sigma_{b}$ represents stress (in MPa) of the femoral cortical bone tissue, $\mathrm{M}$ represents the maximum applied bending moment (in $\mathrm{N} \bullet \mathrm{mm}$ ), y represents the vertical distance from the bone axis to the failure surface (in $\mathrm{mm}$ ), and I represents the $2 \mathrm{nd}$ moment of inertia (in $\mathrm{mm}^{4}$ ), a geometric property of a beam that defines its resistance to bending. For blunt indentation, the applied compressive stress, $\sigma_{\mathrm{c}}$ is determined by the amount of applied force, F, divided by the cross-sectional area, $\mathrm{A}$, of the $1 \mathrm{~mm}$ diameter indenter tip: $\sigma_{\mathrm{c}}=\mathrm{F} / \mathrm{A}$.

Statistical analysis: All data are represented as average values \pm standard deviation. Statistical comparisons between WT and Camkk2 ${ }^{-/}$at three months were by Student's t-test. Statistical comparisons between saline (WT) and STO609 treated samples and Camkk2 ${ }^{-/}$samples were by single factor ANOVA and post-hoc $t$-test. Differences with $p$-value $<0.05$ were deemed significant.

\section{Results}

\section{Trabecular bone microarchitecture in young male mice lacking CaMKK2}

As a first step towards understanding whether the lack of CaMKK2 enhanced bone quality, we analyzed the microarchitecture of the distal femurs from relatively young twelve week old male WT and Camkk2/- mice ( $n=10$ each) using micro-computed tomography ( $\square \mathrm{CT})$ (Figure 1A). As was observed previously in eight week old female mice [55], twelve week old male mice lacking CaMKK2 possessed a significant two-fold higher trabecular bone volume fraction 
compared to age and sex matched WT mice (Figure 1B). Next we analyzed trabecular separation (Tr.Sp) and thickness (Tr.Th) as these are key parameters characterizing the microarchitecture and quality of the trabecular bone, and are indicative of its weight bearing strength [35]. Accordingly, Tr.Sp was 53\% lower in twelve week old male Camkk2 $2^{-/}$mice compared to WT (Figure 1C). Further, trabeculae in the distal femurs of Camkk2 ${ }^{-/}$mice were $30 \%$ thicker than those in age and sex matched WT mice (Figure 1D). Taken together, these data indicate significantly higher trabecular bone volume and architectural quality in the long bones of young male Camkk2/- mice.

\section{Trabecular bone strength in three month-old male Camkk2 ${ }^{-/}$mice}

Ultrastructural features of trabecular bone are optimized by bone remodeling, a process characterized by osteoclast-induced bone resorption and osteoblast-mediated bone formation [66]. Complete inhibition of osteoclast activity results in higher bone volume, but leads to stiff and brittle bones that break or fracture more easily [28, 32]. On the other hand, higher bone volumes and stronger bone can be achieved through increased bone formation by stimulation of osteoblasts without necessarily completely inhibiting osteoclasts $[28,32]$. The lack of CaMKK2 in the present model favors osteoblasts, but the Camkk2 $2^{-/}$long bones do possess osteoclasts at significantly decreased levels compared to WT [55]. Therefore, we surmised that the trabecular bone in the mutant mice is strong with superior biomechanical properties because the continuing, albeit diminished, osteoclast activity prevents over-mineralization and brittleness. 
To test this idea, we performed a blunt indentation test of the trabecular bone of distal femurs from three month or twelve week old male WT and Camkk2 $2^{-/}$mice. Briefly, the thickness of the epiphysis and distance to the base of the epiphyseal plate was measured for individual bones using $\square \mathrm{CT}$ image analysis. This information was employed to trim the distal femurs to expose the epiphyseal plates for precise indentation (Figure 2A). The flat $1 \mathrm{~mm}$ diameter indenter tip produced approximately 1.27 MPa of compressive/contact stress for every $1 \mathrm{~N}$ of force applied. The direct blunt indentation strength of trabecular bone was a significant $62 \%$ higher for Camkk2 $^{-/}(51.2 \mathrm{MPa})$ than that of WT (31.7 MPa) mice (Figure 2B). Thus, the significant increase in epiphyseal trabecular strength correlated linearly with the enhanced BV/TV and superior trabecular architecture in 3 month-old male Camkk2 $2^{--}$mice, in comparison to WT (Figures 1-2), indicating that the global deletion of CaMKK2 contributes to higher bone mass and enhanced strength.

\section{Cortical bone strength in young Camkk2 ${ }^{-/}$male mice}

We next assessed the cortical bone strength by three-point-bending to failure. As shown in Figure 3A, femoral mid-shaft bending strength was increased by a significant $43 \%$ in Camkk2 $^{-/}$mice $(59.4 \pm 8.3 \mathrm{~N} \cdot \mathrm{mm})$ than that in WT $(41.6 \pm 5.7 \mathrm{~N} \bullet \mathrm{mm})($ Figure $3 \mathrm{~A})$. The Camkk2 ${ }^{-/}$femurs are $8 \%$ longer and their mid-shaft diameters are on average 10\% wider than WT (Figures 3C-D). When the differences in cross-sectional area moments of inertia (MOI) between Camkk2 $2^{-/}\left(0.232 \pm 0.044 \mathrm{~mm}^{4}\right)$ and WT mice $\left(0.157 \pm 0.0345 \mathrm{~mm}^{4}\right)$ were considered, the cortical bone tissue bending stress was not significantly different 
between Camkk2 $2^{-/}(129 \pm 30 \mathrm{MPa})$ and WT $(122 \pm 39 \mathrm{MPa})$ mice (Figure 3B-C). Moreover, the similarity in cortical bone thickness between WT $(0.46 \pm 0.05 \mathrm{~mm})$ and Camkk2 ${ }^{-/}(0.5 \pm 0.04 \mathrm{~mm})$ femurs indicate that the differences in endocortical resorption is offset by increased periosteal apposition in the knockout (KO) (Figure 3C). Overall, these data indicate that at twelve weeks of age, the cortical bones in Camkk2 ${ }^{-/}$mice are stronger than WT.

\section{ST0-609 stimulates bone mass accrual in thirty-two week old male mice}

An important question arising from these studies is whether an acute inhibition of CaMKK2 will trigger new bone formation in adult WT male mice at an age where their trabecular bone mass has begun to decline (compare Figures $1 \mathrm{~A}$ and 5A). To this end, we injected thirty-two week old male WT mice with saline or $10 \square$ M STO-609 for 6 weeks and metabolically labeled the newly formed bone with calcein and alizarin red, 7 and 2 days respectively, before the end of the treatment. STO-609 treatment results in markedly elevated calcein and alizarin red incorporation (Figure 4A), indicating enhanced bone formation. Moreover, dynamic histomorphometry measurements indicate significant increases in mineral apposition rate (MAR; $+13 \%$ ) and bone formation rate (BFR; +40\%) following the acute pharmacological inhibition of CaMKK2 in thirty week old mice (Figure 4B-C). Thus, treatment with STO-609 stimulates osteoblast-mediated bone formation in the long bones of adult mice.

We next evaluated bone volume and trabecular microarchitecture in thirtytwo week old saline and STO-609 treated WT as well as Camkk2 ${ }^{--}$mice by $\square$ CT 
analysis. Compared to the twelve week old male cohorts, the thirty-two week old male WT and Camkk2 ${ }^{-/}$mice suffered an $11 \%$ decline in BV/TV (Compare

Figures 1B and 5B). However, the bone volume in the older Camkk2 ${ }^{-/-}$mice was still significantly higher (2-fold) than that in age and sex matched WT (Figures $5 A-B)$, suggesting that the enhanced trabecular bone formation in the $\mathrm{KO}$ mice counteracts the age-dependent bone loss. Moreover, a six week regimen of STO-609 administration in the WT mice resulted in a significant $52.5 \%$ increase in trabecular bone volume fraction (BV/TV) compared to saline treated controls (Figure 5B). Further, parameters determining trabecular quality were significantly higher in older Camkk2 ${ }^{-/}$mice, as trabecular separation was $49 \%$ lower and trabecular thickness was 28\% higher than WT (Figures 5C-D). Treatment of adult WT mice with STO-609 resulted in a $28 \%$ reduction in trabecular separation and a $28 \%$ increase in trabecular thickness (Figures 5C-D). Taken together, these data indicate that acute pharmacological inhibition of CaMKK2 prevents age-associated bone loss occurring in adult mice.

\section{Bone strength assessment in STO-609 treated adult mice}

Having established that STO-609 treatment enhances trabecular bone mass accrual and trabecular architecture in adult mice that are in a maintenance or early catabolic phase of bone metabolism, our next question was whether the STO-609 treated bone possesses superior biomechanical properties and higher strength. Direct blunt indentation tests (Figure 2A) were performed to assess the strength of distal femoral trabecular bone from thirty-two week old Camkk2 ${ }^{-/}$as well as saline (WT) and STO-609 treated male mice. Our results indicate that 
compared to control $(24.9 \pm 4.6 \mathrm{MPa})$; STO-609 treated femurs $(37.1 \pm 7.8 \mathrm{MPa})$ required a significant $48.9 \%$ higher force to break the trabecular bone just below the epiphyseal plate by direct-blunt indentation (Figure 6A). Indeed, the breaking force of trabecular bone was still significantly higher (+61.4\%) in thirty-two week old Camkk2 $2^{-/}$mice $(40.2 \pm 3.8 \mathrm{MPa})$, indicating that the elevated bone mass and trabecular bone strength is maintained in Camkk2 ${ }^{-/}$mice even as they age (Figure 6A).

We then assessed cortical bone strength in these cohorts by the threepoint-bending to failure, and found a significant increase $(+24.8 \%)$ in femoral mid-shaft bending strength in STO-609 treated mice $(45.2 \pm 6 \mathrm{~N} \cdot \mathrm{mm})$ compared to saline-treated WT controls $(36.4 \pm 7.1 \mathrm{~N} \cdot \mathrm{mm})$ (Figure 6B). Consistent with our observations in younger mice, femurs from thirty-two week old Camkk2/- mice possessed a $22.8 \%$ higher three point bending moment $(44.7 \pm 3.8 \mathrm{~N} \cdot \mathrm{mm})$ than WT controls (Figure 6B). Notably, the mid-shaft mediolateral and dorsoventral inner and outer diameters increased significantly ( $+14 \%$ on average) in STO-609 treated femurs than controls, whereas they were on average $11 \%$ higher in Camkk2 $^{-/}$(Figure 6C). Accordingly, as indicated in Figure 6C, the crosssectional area moments of inertia (MOI) were significantly higher in STO-609treated WT $\left(0.157 \pm 0.03 \mathrm{~mm}^{4}\right)$ and Camkk2 ${ }^{-/}\left(0.17 \pm 0.02 \mathrm{~mm}^{4}\right)$, compared to saline-treated control mice $\left(0.112 \pm 0.03 \mathrm{~mm}^{4}\right)$. In contrast to this increase in the mid-shaft geometry, the cortical bone thickness and three-point-bending stress were similar among all three cohorts analyzed (Figure 6C-D). Taken together, these data indicate that the genetic ablation or acute inhibition of CaMKK2 
results in increased bone mass and higher trabecular and cortical bone strength in young and adult mice.

\section{Discussion}

Based on our previous results indicating elevated bone mass subsequent to enhanced osteoblasts and diminished osteoclasts in mice lacking CaMKK2 [55], we hypothesized that the enhanced bone mass translates into improved architectural and biomechanical properties. In this study, we compared biomechanical properties of the trabecular and cortical bone in Camkk2 $2^{-/}$mice as they aged and asked whether acutely inhibiting CaMKK2 in adult mice that are in a maintenance or early catabolic phase of bone metabolism stimulated formation of new bone with superior quality and strength. Indeed, both twelve and thirtytwo week old Camkk2 ${ }^{-/}$mice possessed significantly higher trabecular bone volume and thickness as well as diminished separation, indicating the maintenance of the improved trabecular volume, microarchitecture and weight bearing capacity with age. Biomechanical analyses show superior trabecular and cortical bone strength as well as enhanced mid-shaft geometry in young and adult mice lacking CaMKK2, compared to WT. In contrast to the higher femoral bending strength in the $\mathrm{KO}$, the cortical thickness and bending stress were similar in both genotypes. Metabolic labeling studies indicate stimulation of bone growth in the long bones of thirty-two week old WT mice following the acute inhibition of CaMKK2 using STO-609. In addition to significantly higher bone volume, the trabecular bone from STO-609 treated mice was thicker with less separation compared to age and sex matched saline-treated control mice. 
Additionally, STO-609 treatment significantly enhanced femoral bending strength as well as the femoral mid-shaft cross-sectional $\mathrm{MOI}$, suggesting periosteal bone apposition. Taken together, these data indicate that whereas genetic ablation of CaMKK2 helps in a sustained maintenance of increased bone mass and trabecular bone strength, a short-term acute inhibition of this kinase reverses bone loss and promotes trabecular bone mass accrual as well as superior trabecular and cortical bone strength in adult mice who are in the beginning stages of age-associated catabolic bone loss. The natural history of the WT bone quality reduction can be observed by comparing the measures of both cortical and cancellous bone from the twelve week old mice (Figures 1-3) to those of the thirty-two week old mice (Figure 5-6).

Our previous report [55] as well as the mechanical testing and imaging data from this study support the evidence that the lack of or inhibition of CaMKK2 causes an upregulation of osteoblasts as well as a downregulation of osteoclasts. Maintenance of optimal bone mass and strength is achieved by the balanced interplay between osteoclasts and osteoblasts that form the bone multicellular unit (BMU) in conjunction with osteoid-embedded osteocytes [6769]. Bone strength is determined by the balance of activity within the BMU. For example, during growth, osteoblast activity exceeds osteoclast resorption, resulting in addition of bone and a net positive balance, whereas this is reversed in adults resulting in more bone resorption than addition and a negative balance [33]. Accordingly, over-active remodeling, as observed during aging and other pathological conditions that impact the bone, leads to incomplete bone filling by 
osteoblasts resulting in excessive porosity, cortical thinning and trabecular disconnection [69]. This results in osteoporosis and mechanical flaws within the bone structure; diminishing its strength and rendering it more prone to fragility fractures $[70,71]$.

On one hand, administration of antiresorptive therapies will inhibit ageinduced bone loss and the accumulation of mechanical flaws. On the other hand, complete inhibition of osteoclast activity with or without accompanying anabolic activity will result in brittle bones, such as in osteopetrosis, that are prone to cracking [28]. The most ideal anti-osteoporosis therapies will stimulate osteoblast-mediated anabolic activity while suppressing, but not completely eliminating osteoclasts. In mature or older individuals experiencing active remodeling, such a therapy will create a positive BMU balance by stimulating the formation of new bone with superior architecture and biomechanical properties. Our imaging data show that when CaMKK2 is either absent or inhibited by the application of STO-609, the trabecular bone has a greater volume fraction, greater thickness and less separation. More importantly, administration of STO609 to mature thirty-two week old mice stimulates new bone formation as indicated by dynamic histomorphometry and increased trabecular thickness as evidenced by imaging. Indeed, the STO-609 treated thirty-two week old mice had bone properties similar to twelve week old WT mice. These data clearly suggest a renewed anabolic bone effect in adult mice following an acute pharmacological inhibition of CaMKK2. In Camkk2 $2^{-/-}$mice and STO-609 treated adult mice, this anabolic effect is accompanied by superior trabecular bone 
quality and strength, resulting from the limited, but ongoing osteoclast-mediated remodeling activity which maintains normal bone material properties at the tissue level.

Further, imaging data for the cortical bone of the femoral shaft also shows that there was an anabolic effect on the cortical bone in the KO and STO-609 treated mice since the outer mediolateral and dorsoventral mid-shaft diameters increased significantly compared to age and sex-matched WT mice. Interestingly, the inner diameters also increased, indicating a continued activity of the osteoclasts and endocortical bone resorption. However, increased mid-shaft outer diameters in the $\mathrm{KO}$ and STO-609 treated mice suggest that this is compensated by increased periosteal apposition, resulting in stronger cortices with increased cross-sectional MOI.

The three-point-bending mechanical testing data support this premise because the overall femoral bending strength was shown to be higher in Camkk2 ${ }^{-/}$and STO-609 treated mice, but the tissue level strength was not different. Whereas the structural differences in cortical bone are significant, overall material quality of between the two genotypes is similar, as evidenced by similar cortical mid-shaft thickness and material bending stress. In other words, the net anabolic effects of CaMKK2 genetic ablation or pharmacological inhibition result in significantly higher breaking strength, but the continued remodeling by osteoclasts, albeit reduced in numbers [55], ensure that the overall material quality of the newly formed bone is similar to that in WT. Thus, it is likely that the resulting bone tissue is normal due to continued remodeling, as opposed to 
increased mineralization and brittleness often seen with bisphosphonate therapy and osteopetrosis [72, 73].

In conclusion, the absence or acute inhibition of CaMKK2 represents a model that favors osteoblast-mediated bone formation while suppressing osteoclast activity [55], resulting in increased trabecular bone volume fraction and better cortical midshaft geometry. The accompanying reduced level of osteoclast-mediated remodeling activity imparts the newly formed bone with superior microarchitecture as well as better cancellous compressive and cortical bending strength. The increased bone volume fraction, trabecular bone quality and strength as well as cortical bone strength and mid-shaft cross-sectional area $\mathrm{MOI}$ are sustained in Camkk2/- mice as they matured to a maintenance or early catabolic phase of bone metabolism. More importantly, the fact that STO-609 administration stimulated osteoblast-mediated bone formation in mature, thirtytwo week old mice, demonstrates the potential utility of therapeutically inhibiting CaMKK2 to reverse bone loss while improving cortical and trabecular bone strength in patients with age related bone loss or osteoporosis. 


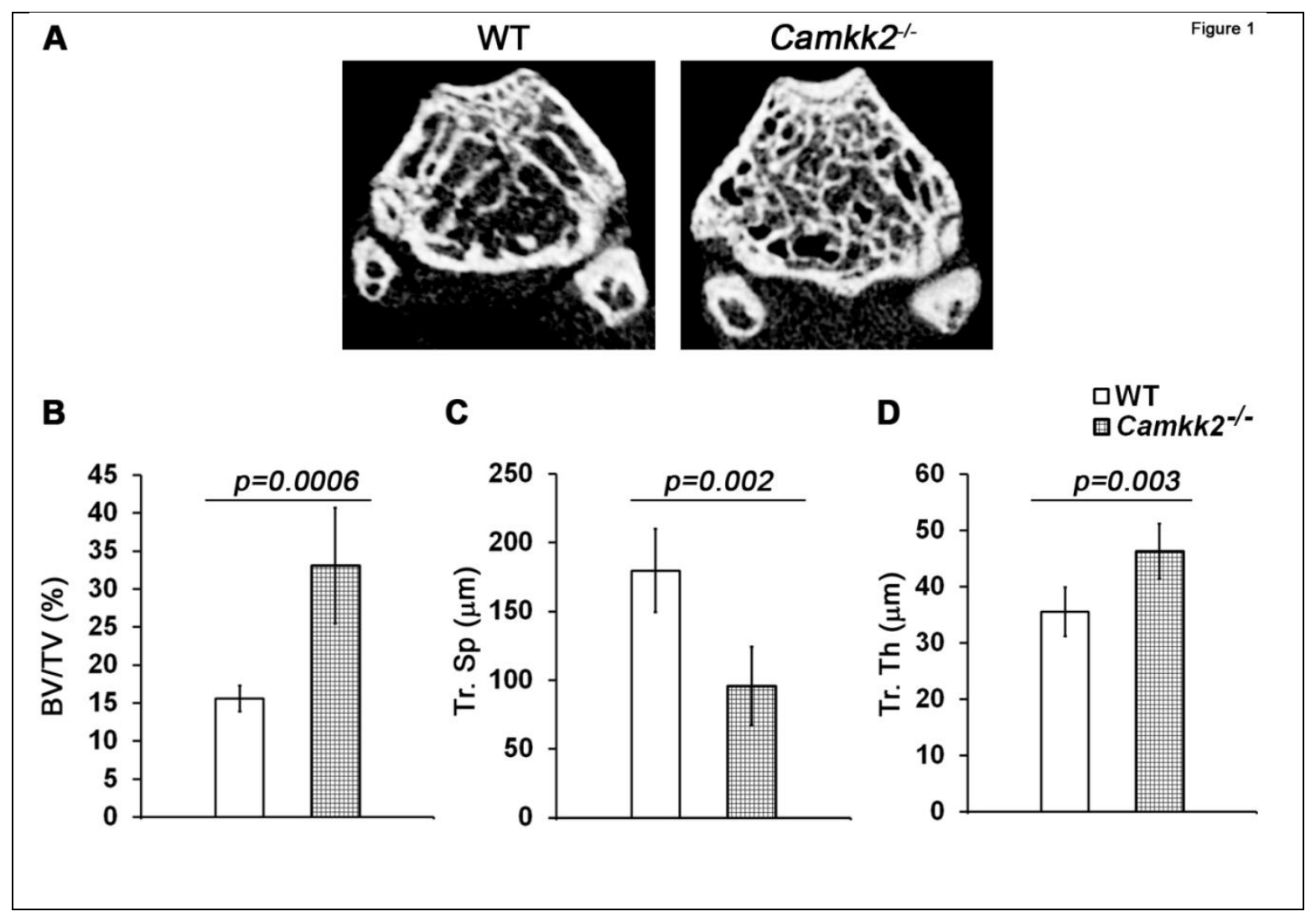

Figure 1. Twelve week old male Camkk2 ${ }^{-/}$mice possess elevated trabecular bone volume fracture and improved microarchitecture. (A) Digital $\mu C T$ crosssectional images of distal femurs showing enhanced trabecular bone in Camkk2 $2^{-/}$ mice compared to age and sex-matched WT. Representative images from $n=10$ each are shown. (B-D) Average $\mu$ CT measurements of BV/TV (\%), Tr. Sp. ( $\mu \mathrm{m})$ and Tr. Th. $(\mu \mathrm{m})$ parameters from distal femurs of twelve week old male WT and Camkk2 $2^{-/}$mice $(\mathrm{n}=10$ each) are shown. Differences with $p$-values $<0.05$ compared to WT were deemed significant. 
A

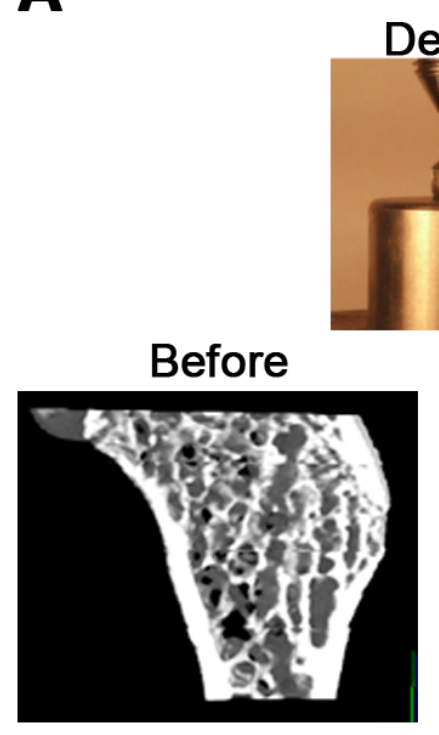

B $\square W T$

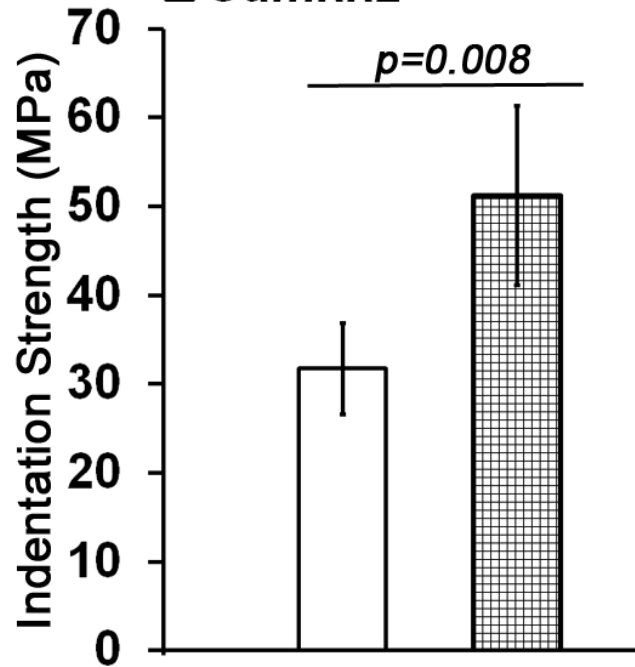

Figure 2. Higher trabecular bone strength in younger Camkk2 ${ }^{-/}$mice. (A) (Top) Digital photograph showing the direct blunt indentation device comprising of a flat $1 \mathrm{~mm}$ diameter indenter tip positioned above the distal femur mounted on a \#10 cap screw head. (Bottom) Digital $\mu \mathrm{CT}$ images of the epiphyseal region taken of the same distal femur before and after direct blunt indentation. Average direct blunt indentation strength \pm standard deviation of the trabecular bone from WT and Camkk2 $2^{-/}$mice ( $\mathrm{n}=10$ each) calculated in MPa is shown. The difference in trabecular bone strength between WT and Camkk2 ${ }^{-/}$distal femurs is significant with a $p$-value of 0.008 . 
A

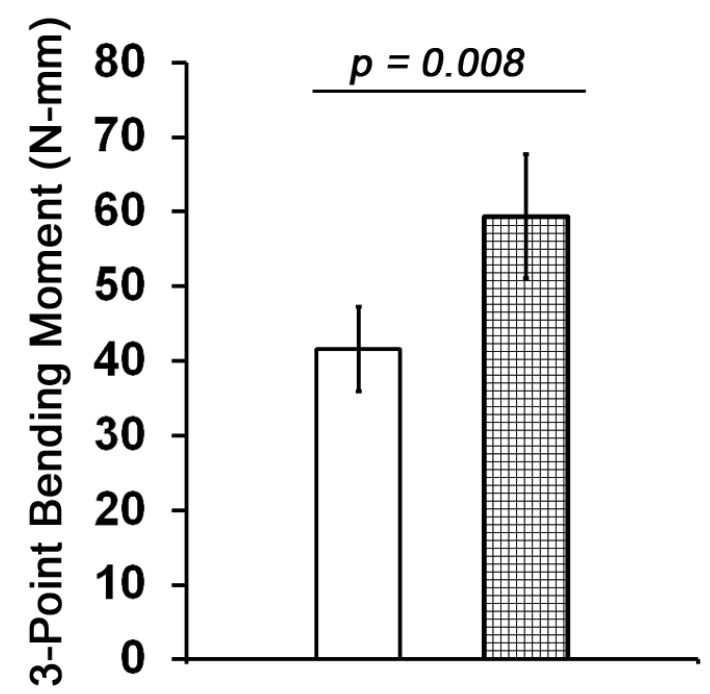

C

\begin{tabular}{lll}
\hline & \multicolumn{2}{l}{ Femur mid-diaphysis } \\
\cline { 2 - 3 } & WT & Camkk2 $^{-/}$ \\
\hline Mediolateral inner diameter $(\mathrm{mm})$ & $1.33 \pm 0.05$ & $1.5 \pm 0.1^{*}$ \\
Mediolateral outer diameter $(\mathrm{mm})$ & $1.84 \pm 0.05$ & $2.0 \pm 0.14^{*}$ \\
Dorsoventral inner diameter $(\mathrm{mm})$ & $0.87 \pm 0.04$ & $1 \pm 0.03^{*}$ \\
Dorsoventral outer diameter $(\mathrm{mm})$ & $1.3 \pm 0.07$ & $1.5 \pm 0.03^{*}$ \\
Crosssectional area moment of & $0.157 \pm 0.04$ & $0.232 \pm 0.04^{*}$ \\
inertia $\left(\mathrm{mm}^{4}\right)$ & $0.46 \pm 0.05$ & $0.5 \pm 0.04$ \\
Cortical bone thickness $(\mathrm{mm})$ & &
\end{tabular}

B

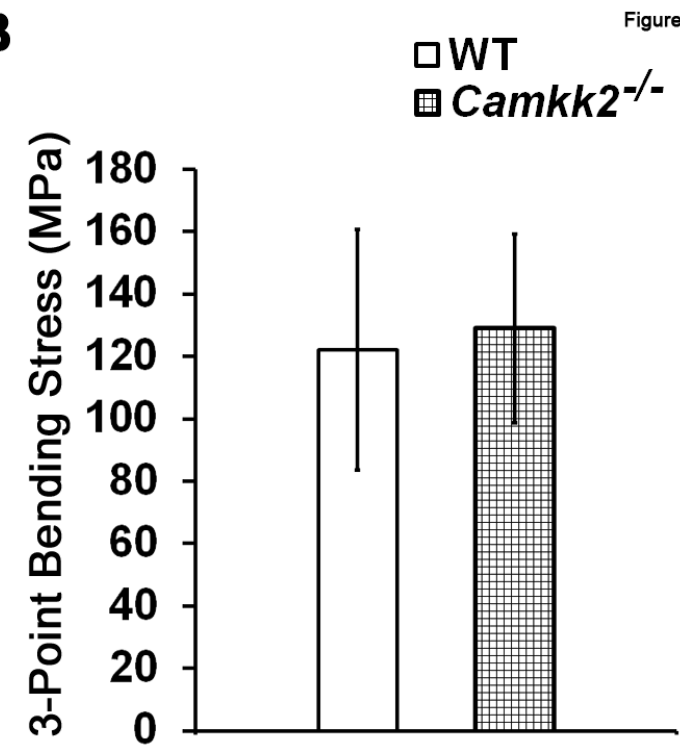

D

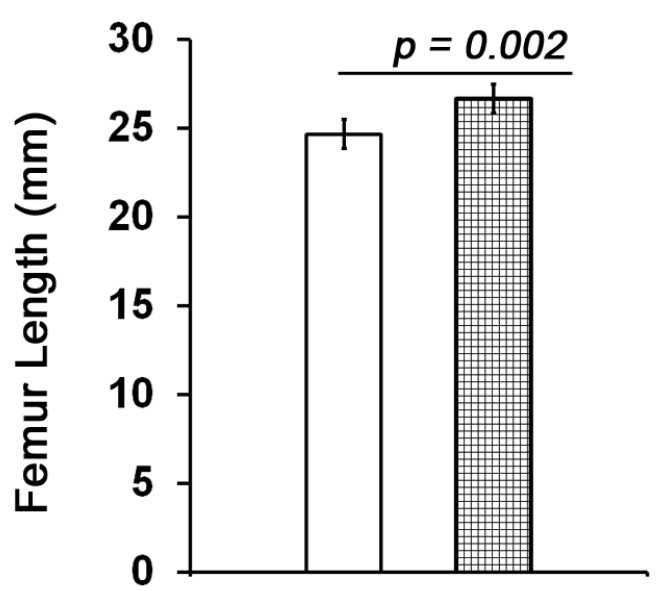


Figure 3. Lack of CaMKK2 results in increased cortical bone bending strength and mid-diaphysis geometry without affecting bending stress. Graphs depicting average three-point (A) bending moment (N.mm) and (B) bending stress $(\mathrm{MPa}) \pm$ standard deviation from WT and Camkk2 ${ }^{-/}$mice $(n=10$ each). Camkk2 ${ }^{-/-}$femurs possess a significantly higher bending moment ( $p$ value $=0.008$ ), compared to $\mathrm{WT}$, but the bending stress is similar between the two genotypes. (C) Table depicting femur mid-shaft parameters measured by $\mu C T$ imaging. Average values \pm standard deviation from WT and Camkk2 $2^{-/}$mice $\left(n=10\right.$ each) are shown. ${ }^{*}$ denotes where the difference in these parameters between the two genotypes is statistically significant with a $p$-value $<0.03$. (D) Average femur length from WT and Camkk2 ${ }^{-/}$mice ( $\mathrm{n}=10$ each) is shown. $p$ value $=0.002$ 
A

Control

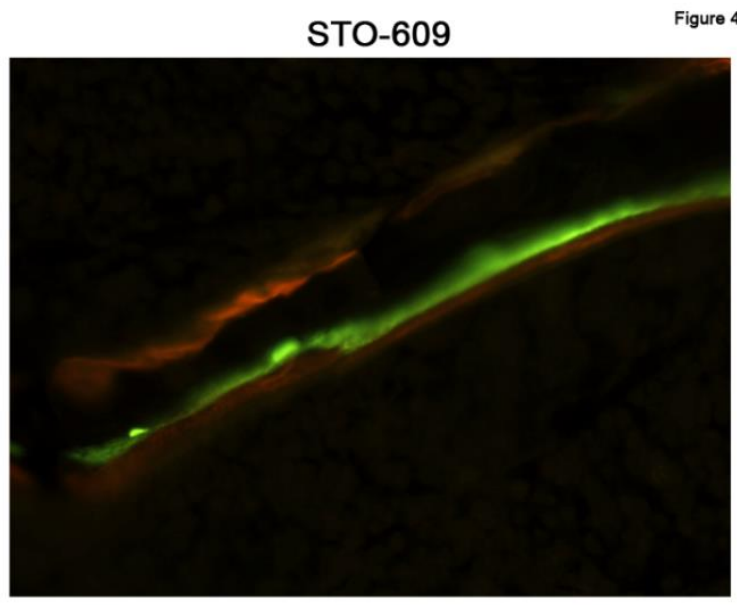

B
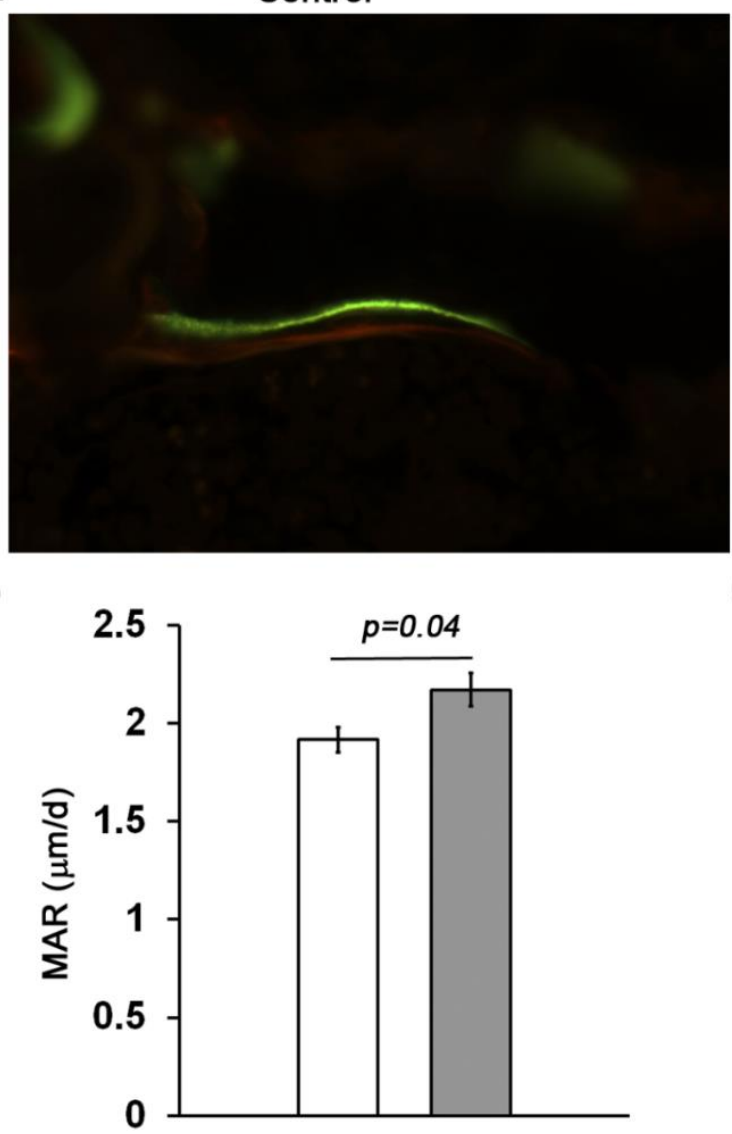

C

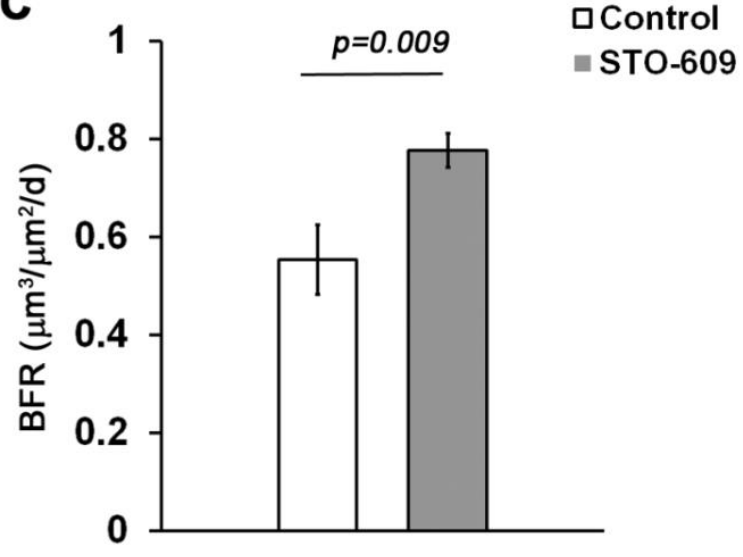

Figure 4. STO-609 administration stimulates trabecular bone formation in thirty-two week old adult male mice. (A) Representative calcein and alizarin red-labelled sections of proximal tibiae from thirty-two week old male mice that were administered saline or STO-609 for six weeks (400X magnification). (B-C) Dynamic histomorphometry analysis of trabecular bone formation in proximal tibiae: mineral apposition rate (MAR) and bone formation rate (BFR). Average values \pm standard deviation from saline $(n=5)$ and STO-609 treated $(n=8)$ samples are shown. Differences with $p$-values $<0.05$ were deemed significant. 
A

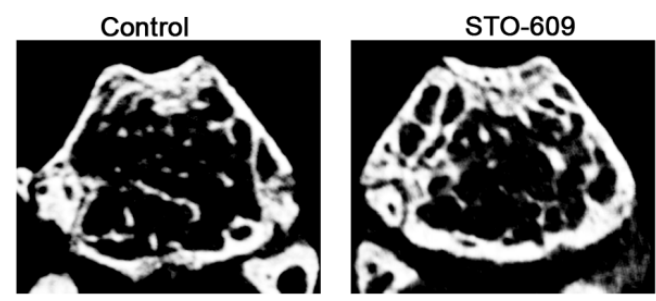

B

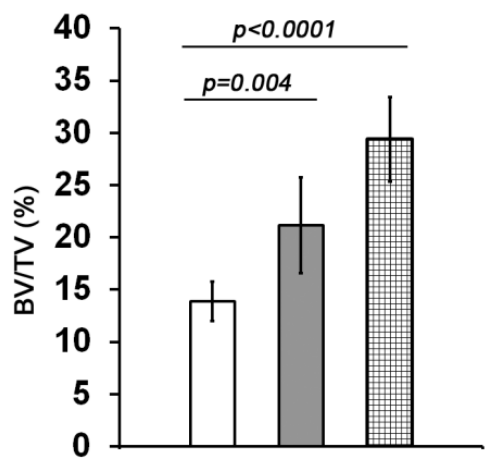

C

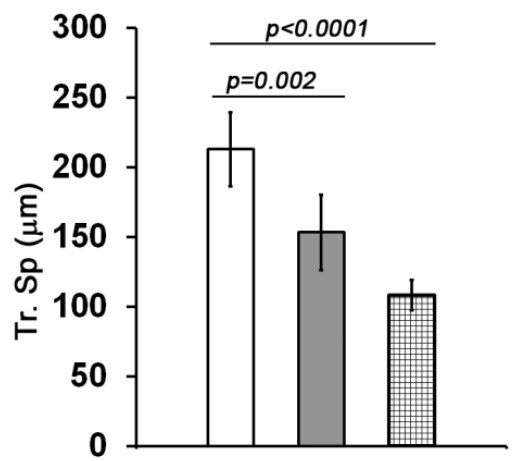

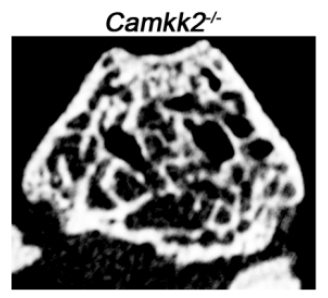

D

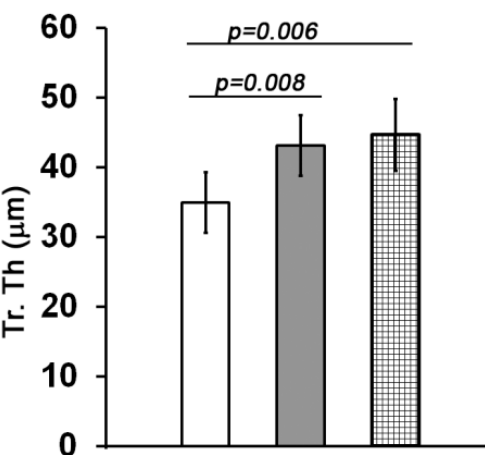

Figure 5. Increased trabecular bone volume and enhanced microarchitecture is sustained in adult mice lacking or acutely inhibited for CaMKK2. (A) Representative digital $\mu \mathrm{CT}$ cross-sectional images of distal femurs showing enhanced trabecular bone in thirty-two week old week old male Camkk2 $^{-/}(n=5)$ and STO-609 treated $(n=11)$ mice compared to age and sexmatched saline-treated WT control mice $(n=6)$. Mice were treated for six weeks with STO-609. (B-D) Average ( \pm standard deviation) $\mu \mathrm{CT}$ measurements of BV/TV (\%), Tr. Sp. $(\mu \mathrm{m})$ and Tr. Th. $(\mu \mathrm{m})$ parameters from distal femurs of adult Camkk2 $2^{-}$as well as STO and saline-treated WT mice are shown. Differences with $p$-values $<0.05$ compared to WT were deemed significant. 


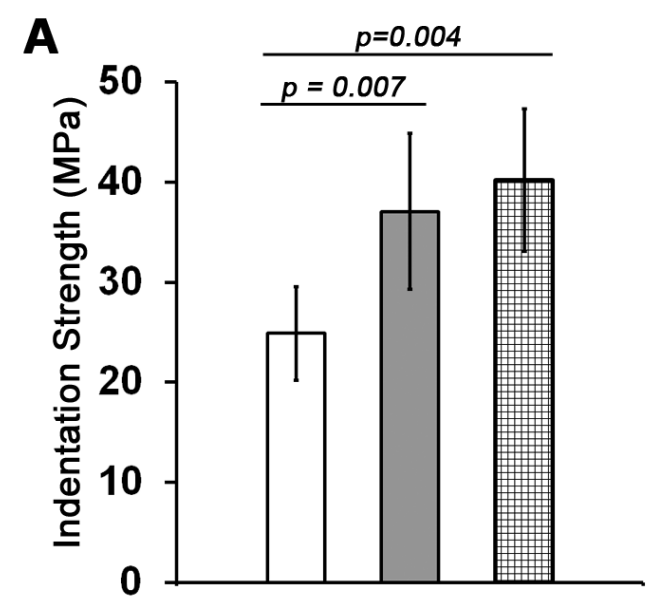

C

Figure 6

B

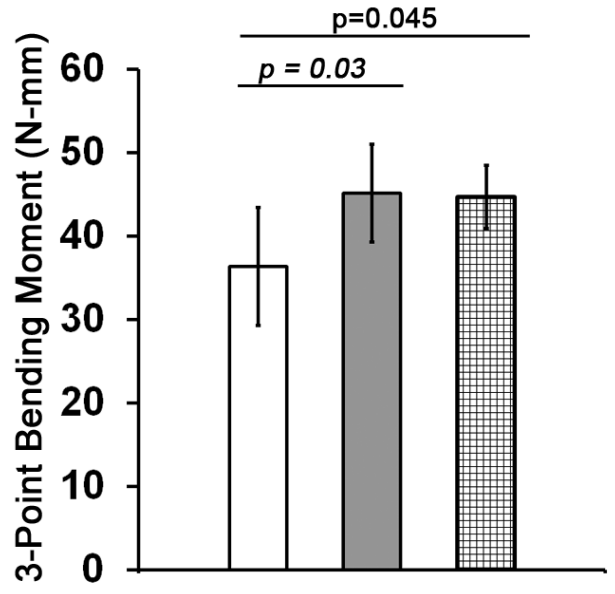


Figure 6. Higher trabecular and cortical bone strength in adult Camkk2 ${ }^{-/-}$ and STO-609-treated WT mice. (A) Average direct blunt indentation strength \pm standard (MPa) of the trabecular bone from thirty-two week old Camkk2 ${ }^{-/}(n=5)$ as well as saline $(n=6)$ and STO-609-treated mice $(n=11)$ calculated in MPa is shown. Trabecular blunt indentation strength of Camkk2 ${ }^{-/}(p=0.004)$ and STO609-treated $(p=0.007)$ distal femurs were significantly higher than that of WTcontrol. (B) Average 3-point bending moment ( $\mathrm{N}-\mathrm{mm})$ of femurs from indicated genotypes are shown. Differences with $p$-values $<0.05$ were deemed significant. (C) Table showing femur mid-shaft parameters measured by $\mu \mathrm{CT}$ imaging showing average values \pm standard deviation from 32 week old Camkk2 $^{-/}(n=5)$ as well as saline $(n=6)$ and STO-609-treated mice $(n=11) .{ }^{*}$ and @ denote where the differences with the saline treated WT controls are statistically significant with a $p$-value $<0.05$. (D) Average three-point bending stress values \pm standard deviation (MPa) from adult $\operatorname{Camkk2}^{-/}(\mathrm{n}=5)$ as well as saline $(n=6)$ and STO-609-treated mice $(n=11)$ are depicted. Bending stress was similar among all three genotypes. 


\section{CHAPTER 4}

\section{SUMMARY AND DISCUSSION}

Osteoporosis is pathological condition that can be treated in a number of ways, such as exercise [6] changes in diet [8], and/or by pharmacological intervention [10-12]. However, pharmacological therapies that are currently available primarily block bone resorption without inducing the replacement of lost bone. The next generation anti-osteoporosis therapies will stimulate new bone formation while inhibiting excessive bone resorption. Such a therapy will be highly desirable as it will improve bone quantity and quality in patients, lowering their risk for fragility fractures. Previous studies from our laboratory recently demonstrated that the genetic ablation or pharmacological inhibition of CaMKK2 results in a net increase in bone mass in mice [20]. Mice deficient in CaMKK2 show an increase in osteoblasts concurrent with a decrease in osteoclasts, resulting in enhanced trabecular bone volume [20]. The goal of my thesis research project was to investigate whether the enhanced bone volume and microarchitecture translated into superior strength. It was hypothesized, as outlined in Chapter 3, that the increased amount of bone mass would lead to increase in bone quality and strength. However, very limited numbers of techniques were available to effectively test trabecular bone strength of mouse 
long bones. Hence to enable us test this hypothesis, we developed an improved technique to test murine trabecular bone strength and this is described in Chapter 2.

With micro-CT imaging mechanical properties of bone can be predicted. This predictable quality of bone was utilized in developing an improved method to prepare the tissue for distal femur indentation. With a murine genetic model that has a predictable difference in microarchitecture; it was possible to refine this method to observe mechanical differences. Indeed, with removal of the epiphysis at the epiphyseal/metaphyseal junction, it was possible to test the hypothesis that increased amounts of trabecular bone lead to increased trabecular bone strength. Chapter 2 concludes that indentation of the distal femur can accurately depict the correlation between bone volume fraction and bone strength. This increase showed a strong $r^{2}$ coefficient of 0.654 . With the resulting success of the technique developed in Chapter 2, it was then possible to test whether or not the increased mass of bone lead to superior architecture and strength. Overall Camkk2 $^{--}$and STO-609-treated animals showed increased bone quality and strength over wild type cohorts. In conclusion with the evidence gathered, this thesis shows that the absence or pharmacological inhibition of CaMKK2 positively affects bone growth, microarchitecture and strength. 


\section{REFERENCES}

1. O'Brien, C., et al., Glucocorticoids act directly on osteoblasts and osteocytes to induce their apoptosis and reduce bone formation and strength. Endocrinology, 2004. 145(4): p. 1835-1841.

2. Weinstein, R., Clinical practice. Glucocorticoid-induced bone disease. The New England journal of medicine, 2011. 365(1): p. 62-70.

3. van Staa, T., et al., Epidemiology of fractures in England and Wales. Bone, 2001. 29(6): p. 517-522.

4. Leboime, A., et al., Osteoporosis and mortality. Joint, bone, spine : revue du rhumatisme, 2010. 77 Suppl 2: p. 12.

5. Alliston, T., Biological Regulation of Bone Quality. Current osteoporosis reports, 2014.

6. Bass, S., P. Eser, and R. Daly, The effect of exercise and nutrition on the mechanostat. Journal of musculoskeletal \& neuronal interactions, 2005. 5(3): p. 239-254.

7. Karlsson, M., et al., Prevention of falls in the elderly: a review. 2013.

8. Kalkwarf, H., J. Khoury, and B. Lanphear, Milk intake during childhood and adolescence, adult bone density, and osteoporotic fractures in US women. The American journal of clinical nutrition, 2003. 77(1): p. 257-265. 
9. Tang, B., et al., Use of calcium or calcium in combination with vitamin $D$ supplementation to prevent fractures and bone loss in people aged 50 years and older: a meta-analysis. Lancet, 2007. 370(9588): p. 657-666.

10. Russell, R.G., et al., Mechanisms of action of bisphosphonates:

similarities and differences and their potential influence on clinical efficacy. Osteoporosis international : a journal established as result of cooperation between the European Foundation for Osteoporosis and the National Osteoporosis Foundation of the USA, 2008. 19(6): p. 733-759.

11. Narayanan, P., Denosumab: A comprehensive review. South Asian journal of cancer, 2013. 2(4): p. 272-277.

12. Blick, S., S. Dhillon, and S. Keam, Teriparatide: a review of its use in osteoporosis. Drugs, 2008. 68(18): p. 2709-2737.

13. Cramer, J., et al., The effect of dosing frequency on compliance and persistence with bisphosphonate therapy in postmenopausal women: a comparison of studies in the United States, the United Kingdom, and France. Clinical therapeutics, 2006. 28(10): p. 1686-1694.

14. Anderson, K., et al., Hypothalamic CaMKK2 contributes to the regulation of energy balance. Cell metabolism, 2008. 7(5): p. 377-388.

15. Racioppi, L. and A. Means, Calcium/calmodulin-dependent protein kinase kinase 2: roles in signaling and pathophysiology. The Journal of biological chemistry, 2012. 287(38): p. 31658-31665. 
16. Mizuno, K., et al., Calcium/calmodulin kinase kinase beta has a malespecific role in memory formation. Neuroscience, 2007. 145(2): p. 393402.

17. Luo, X.J., et al., Convergent lines of evidence support CAMKK2 as a schizophrenia susceptibility gene. Molecular psychiatry, 2013.

18. Teng, E., L. Racioppi, and A. Means, A cell-intrinsic role for CaMKK2 in granulocyte lineage commitment and differentiation. Journal of leukocyte biology, 2011. 90(5): p. 897-909.

19. Kukimoto-Niino, M., et al., Crystal structure of the $\mathrm{Ca}^{2+} /$ calmodulindependent protein kinase kinase in complex with the inhibitor STO-609. The Journal of biological chemistry, 2011. 286(25): p. 22570-22579.

20. Cary, R., et al., Inhibition of $\mathrm{Ca}^{2+} /$ calmodulin-dependent protein kinase kinase 2 stimulates osteoblast formation and inhibits osteoclast differentiation. Journal of bone and mineral research : the official journal of the American Society for Bone and Mineral Research, 2013. 28(7): p. $1599-1610$.

21. Seeman, E., Bone quality: the material and structural basis of bone strength. Journal of bone and mineral metabolism, 2007. 26(1): p. 1-8.

22. Doube, M., et al., Trabecular bone scales allometrically in mammals and birds. Proceedings. Biological sciences / The Royal Society, 2011. 278(1721): p. 3067-3073.

23. Turner, C.H. and D.B. Burr, Basic biomechanical measurements of bone: a tutorial. Bone, 1992. 14(4): p. 595-608. 
24. Turner, C.H., Bone strength: current concepts. Annals of the New York Academy of Sciences, 2006. 1068: p. 429-446.

25. Seeman, E. and P.D. Delmas, Bone quality--the material and structural basis of bone strength and fragility. The New England journal of medicine, 2006. 354(21): p. 2250-2261.

26. Pressel, T., et al., Mechanical properties of femoral trabecular bone in dogs. Biomedical engineering online, 2004. 4: p. 17.

27. Cary, R.L., et al., Inhibition of $\mathrm{Ca}^{2+} /$ calmodulin-dependent protein kinase kinase 2 stimulates osteoblast formation and inhibits osteoclast differentiation. Journal of bone and mineral research : the official journal of the American Society for Bone and Mineral Research, 2013. 28(7): p. 1599-1610.

28. Turner, C.H., Bone strength: current concepts. Ann N Y Acad Sci, 2006. 1068: p. 429-46.

29. Duan, Y., et al., Structural and biomechanical basis of sexual dimorphism in femoral neck fragility has its origins in growth and aging. J Bone Miner Res, 2003. 18(10): p. 1766-74.

30. Filardi, S., et al., Femoral neck fragility in women has its structural and biomechanical basis established by periosteal modeling during growth and endocortical remodeling during aging. Osteoporos Int, 2004. 15(2): p. 1037.

31. Fields, A.J. and T.M. Keaveny, Trabecular architecture and vertebral fragility in osteoporosis. Curr Osteoporos Rep, 2012. 10(2): p. 132-40. 
32. Seeman, E., Bone quality: the material and structural basis of bone strength. J Bone Miner Metab, 2008. 26(1): p. 1-8.

33. Seeman, E. and P.D. Delmas, Bone quality--the material and structural basis of bone strength and fragility. N Engl J Med, 2006. 354(21): p. 225061.

34. Silva, B.C., et al., Osteoporosis: what's new and on the horizon. Clin Obstet Gynecol, 2013. 56(4): p. 730-8.

35. Khosla, S., Pathogenesis of age-related bone loss in humans. J Gerontol A Biol Sci Med Sci, 2013. 68(10): p. 1226-35.

36. Long, F. and D.M. Ornitz, Development of the endochondral skeleton. Cold Spring Harb Perspect Biol, 2013. 5(1): p. a008334.

37. Manolagas, S.C., T. Bellido, and R.L. Jilka, New insights into the cellular, biochemical, and molecular basis of postmenopausal and senile osteoporosis: roles of IL-6 and gp130. Int J Immunopharmacol, 1995. 17(2): p. 109-16.

38. Manolagas, S.C., T. Bellido, and R.L. Jilka, Sex steroids, cytokines and the bone marrow: new concepts on the pathogenesis of osteoporosis. Ciba Found Symp, 1995. 191: p. 187-96; discussion 197-202.

39. Xu, X.H., et al., Molecular genetic studies of gene identification for osteoporosis: the 2009 update. Endocr Rev, 2010. 31(4): p. 447-505.

40. Bliziotes, M., et al., Reduced G-protein-coupled-receptor kinase 2 activity results in impairment of osteoblast function. Bone, 2000. 27(3): p. 367-73. 
41. Bliziotes, M., et al., Bone histomorphometric and biomechanical abnormalities in mice homozygous for deletion of the dopamine transporter gene. Bone, 2000. 26(1): p. 15-9.

42. Globus, R.K., et al., Skeletal phenotype of growing transgenic mice that express a function-perturbing form of beta1 integrin in osteoblasts. Calcif Tissue Int, 2005. 76(1): p. 39-49.

43. Hawse, J.R., et al., TIEG-null mice display an osteopenic gender-specific phenotype. Bone, 2008. 42(6): p. 1025-31.

44. Hawse, J.R., et al., Estrogen receptor beta isoform-specific induction of transforming growth factor beta-inducible early gene-1 in human osteoblast cells: an essential role for the activation function 1 domain. Mol Endocrinol, 2008. 22(7): p. 1579-95.

45. Jeyabalan, J., et al., Mice lacking AMP-activated protein kinase alpha1 catalytic subunit have increased bone remodelling and modified skeletal responses to hormonal challenges induced by ovariectomy and intermittent PTH treatment. J Endocrinol, 2012. 214(3): p. 349-58.

46. Mun, S.H., et al., Deletion of CD74, a putative MIF receptor, in mice enhances osteoclastogenesis and decreases bone mass. J Bone Miner Res, 2013. 28(4): p. 948-59.

47. Pennypacker, B., et al., Bone density, strength, and formation in adult cathepsin K (-/-) mice. Bone, 2009. 44(2): p. 199-207. 
48. Lloyd, S.A., et al., Connexin 43 deficiency attenuates loss of trabecular bone and prevents suppression of cortical bone formation during unloading. J Bone Miner Res, 2012. 27(11): p. 2359-72.

49. Wattanachanya, L., et al., Increased bone mass in mice lacking the adipokine apelin. Endocrinology, 2013. 154(6): p. 2069-80.

50. Mao, D., et al., PLCY2 regulates osteoclastogenesis via its interaction with ITAM proteins and GAB2. J Clin Invest, 2006. 116(11): p. 2869-2879.

51. Vaira, S., et al., RelB is the NF-KB subunit downstream of NIK responsible for osteoclast differentiation. Proceedings of the National Academy of Sciences, 2008. 105(10): p. 3897-3902.

52. Robling, A.G., et al., Anabolic and Catabolic Regimens of Human Parathyroid Hormone 1-34 Elicit Bone- and Envelope-Specific Attenuation of Skeletal Effects in Sost-Deficient Mice. Endocrinology, 2011. 152(8): p. 2963-2975.

53. Joeng, K.S. and F. Long, Constitutive activation of Gli2 impairs bone formation in postnatal growing mice. PLoS ONE, 2013. 8(1): p. e55134.

54. Dela Cruz, A., et al., Reduced trabecular bone mass and strength in mice overexpressing Galpha11 protein in cells of the osteoblast lineage. Bone, 2014. 59: p. 211-22.

55. Cary, R.L., et al., Inhibition of ca(2+) /calmodulin-dependent protein kinase kinase 2 stimulates osteoblast formation and inhibits osteoclast differentiation. J Bone Miner Res, 2013. 28(7): p. 1599-610. 
56. Colomer, J. and A.R. Means, Physiological roles of the Ca2+/CaMdependent protein kinase cascade in health and disease. Subcell Biochem, 2007. 45: p. 169-214.

57. Kitsos, C.M., et al., Calmodulin-dependent protein kinase IV regulates hematopoietic stem cell maintenance. The Journal of biological chemistry, 2005. 280(39): p. 33101-8.

58. Kokubo, M., et al., BDNF-mediated cerebellar granule cell development is impaired in mice null for CaMKK2 or CaMKIV. J Neurosci, 2009. 29(28): p. 8901-13.

59. Anderson, K.A., et al., Hypothalamic CaMKK2 Contributes to the Regulation of Energy Balance. Cell Metabolism, 2008. 7(5): p. 377-388.

60. Racioppi, L. and A.R. Means, Calcium/Calmodulin-Dependent Protein Kinase Kinase 2: Roles in Signaling and Pathophysiology. J Biol Chem, 2012.

61. Racioppi, L., et al., Calcium/Calmodulin-dependent Protein Kinase Kinase 2 Regulates Macrophage-mediated Inflammatory Responses. J Biol Chem, 2012. 287(14): p. 11579-91.

62. Anderson, K.A., et al., Deletion of CaMKK2 from the liver lowers blood glucose and improves whole-body glucose tolerance in the mouse. Mol Endocrinol, 2012. 26(2): p. 281-91.

63. Tokumitsu, H., et al., STO-609, a specific inhibitor of the $\mathrm{Ca}(2+) /$ calmodulin-dependent protein kinase kinase. J Biol Chem, 2002. 277(18): p. 15813-8. 
64. Yao, W., et al., Inhibition of the progesterone nuclear receptor during the bone linear growth phase increases peak bone mass in female mice. PLoS One, 2010. 5(7): p. e11410.

65. Parfitt, A.M., et al., Bone histomorphometry: standardization of nomenclature, symbols, and units. Report of the ASBMR Histomorphometry Nomenclature Committee. J Bone Miner Res, 1987. 2(6): p. 595-610.

66. Birkenhager-Frenkel, D.H., et al., Changes of interstitial bone thickness with age in men and women. Bone, 1993. 14(3): p. 211-6.

67. Grimston, S.K., et al., Connexin43 modulates post-natal cortical bone modeling and mechano-responsiveness. Bonekey Rep, 2013. 2: p. 446.

68. Laurent, M., et al., Androgens and estrogens in skeletal sexual dimorphism. Asian J Androl, 2014. 16(2): p. 213-22.

69. Reeve, J. and N. Loveridge, The fragile elderly hip: mechanisms associated with age-related loss of strength and toughness. Bone, 2014. 61: p. $138-48$.

70. Fowlkes, J.L., et al., Osteo-promoting effects of insulin-like growth factor I (IGF-I) in a mouse model of type 1 diabetes. Bone, 2013. 57(1): p. 36-40.

71. Nyman, J.S., et al., Increasing duration of type 1 diabetes perturbs the strength-structure relationship and increases brittleness of bone. Bone, 2011. 48(4): p. 733-40. 
72. Sasaki, $\mathrm{H}$. , et al., Effects of combination treatment with alendronate and vitamin K(2) on bone mineral density and strength in ovariectomized mice. J Bone Miner Metab, 2010. 28(4): p. 403-9.

73. Del Fattore, A., A. Cappariello, and A. Teti, Genetics, pathogenesis and complications of osteopetrosis. Bone, 2008. 42(1): p. 19-29. 


\section{APPENDIX}

CHAPTER 3:

ACUTE INHIBITION OR LACK OF CAMKK2 ENHANCES BONE MASS, MICROARCHITECTURE AND STRENGTH IN ADULT MICE

Zachary J. Pritchard, ${ }^{1}$ Rachel L. Cary, ${ }^{2}$ Yang, Chang, ${ }^{3}$ Deborah V. Novack, ${ }^{3}$ Michael J. Voor ${ }^{4,5,{ }^{*}}$ and Uma Sankar ${ }^{1,2,{ }^{*}}$

${ }^{1}$ Department of Pharmacology and Toxicology, ${ }^{2}$ James Graham Brown Cancer Center and Owensboro Cancer Research Program, University of Louisville School of Medicine, Louisville, KY; ${ }^{3}$ Department of Medicine and Pathology, Washington University School of Medicine, St. Louis, MO; ${ }^{4}$ Department of Orthopaedic Surgery, University of Louisville School of Medicine, Louisville, KY; ${ }^{5}$ Department of Bioengineering, University of Louisville Speed School of Engineering, Louisville, KY.

*Address correspondences to: Uma Sankar, Ph.D., 505 South Hancock Street, 412 CTRB, Louisville, KY 40202; Email: uma.sankar@louisville.edu; or Michael J. Voor, Ph.D., Orthopaedic Bioengineering Laboratory, University of Louisville, 40292 USA. Tel: 502-852-7067, Fax: 502-852-7227, Email:

mike.voor@louisville.edu. 
Running Title: CaMKK2 inhibition increases trabecular and cortical bone strength.

Grant Support: This study was supported in part by grants from the American Cancer Society (RSG 13-301-01), Department of Defense/CDMRP (PR121604) and an intramural competitive enhancement grant from the Office of the Vice President for Research at the University of Louisville to US. DVN is supported by AR052705 (NIAMS). Dynamic histomorphometry and histological analysis was supported by The Center for Musculoskeletal Biology and Medicine at Washington University, P30AR057235 (NIAMS).

\section{Disclosures}

All authors of this manuscript state that they have no conflict of interest. The authors further state that there are no restrictions on full access for all authors to all raw data, statistical analyses and material used in the study reported in this manuscript.

\section{Abstract}

The tissue-restricted multifunctional $\mathrm{Ca}^{2+} /$ calmodulin (CaM)-dependent protein kinase kinase 2 (CaMKK2) has roles in the anabolic and catabolic pathways of bone remodeling. Specifically, genetic ablation of CaMKK2 positively influences osteoblasts and negatively affects osteoclasts, resulting in a net increase of bone mass, and its pharmacological inhibition through STO-609 protects mice from ovariectomy-induced osteoporosis. However, it was not known whether the enhanced bone mass formed in the absence of CaMKK2 
would possess improved architectural and biomechanical properties. Here we analyzed biomechanical properties of the long bones in Camkk2 $2^{-/}$mice as they aged and examined whether acutely inhibiting CaMKK2 in adult mice stimulated formation of new bone with superior quality and strength. Our results indicate that mice lacking CaMKK2 possess increased trabecular bone volume fraction with higher indentation strength and microarchitectural quality. These superior trabecular biomechanical properties as well as increased cortical bone bending strength and mid-shaft cross-sectional area moment of inertia (MOI) are maintained in $\mathrm{Camkk2}^{-/}$mice as they matured to adults. Moreover, acute pharmacological inhibition of CaMKK2 using STO-609 promotes trabecular bone mass accrual as well as improved trabecular and cortical bone strength in adult mice that are otherwise in an early catabolic phase of bone metabolism. The reduced level of osteoclast-mediated remodeling activity present in mice lacking or inhibited for CaMKK2 activity confers the newly formed bone with superior biomechanical qualities. Thus, pharmacological inhibition of CaMKK2 is a potential dual action anabolic/anti-catabolic therapeutic strategy to reverse bone loss while maintaining bone with high quality and strength.

Key words: $\mathrm{Ca}^{2+} /$ calmodulin (CaM)-dependent protein kinase kinase 2, STO609 , bone strength, direct blunt indentation, 3-point bending moment, microarchitecture, osteoblasts, osteoclasts. 


\section{Acknowledgements}

This work was supported in part by grants to US and MJV from the American Cancer Society (RSG 13-301-01), Department of Defense/CDMRP (PR121604) and an intramural competitive enhancement grant from the Office of the Vice President for Research at the University of Louisville. DVN is supported by AR052705 (NIAMS). Dynamic histomorphometry and histological analysis was supported by The Center for Musculoskeletal Biology and Medicine at Washington University, P30AR057235 (NIAMS). 


\section{CURRICULUM VITA}

NAME: $\quad$ Zachary James Pritchard

ADDRESS: 4300 Wilkinson Way, Lovelock Nevada 89419

DOB: $\quad$ Fallon, Nevada December 30, 1988

EDUCATION \& TRAINING:

B.S. Environmental Science

Sierra Nevada College

$2007-2011$

AWARDS: Plenary Poster Award- ASBMR Annual Meeting 2013

Young Investigator Travel Award ASBMR Annual Meeting 2013

PROFESSIONAL SOCIETIES:

American Society of Bone Mineral Research (ASBMR) 2012-2013

PUBLICATIONS:

Pritchard ZJ, Cary RL, Yang, C, Novack DN, Voor MJ, and Sankar U. Acute Inhibition or Lack of CaMKK2 Enhances Bone Mass, Microarchitecture and

Strength in Adult Mice. Manuscript under review at JBMR.

NATIONAL MEETING PRESENTATION

Pritchard Z, Cary R, Novack D, Voor M and Sankar U. CaMKK2 Inhibition as a Novel Bone Anabolic Strategy in the Prevention of Post-Menopausal and Therapy-Induced Osteoporosis. Plenary Poster at the $35^{\text {th }}$ Annual Meeting of 
The American Society for Bone and Mineral Research, October 4-7, 2013, Baltimore, Maryland.

REFEREED JOURNALS

BOOKS AND SYMPOSIA

INVITED PRESENTATIONS 